\title{
Boolean Logic Algebra Driven Similarity Measure for Text Based Applications
}

\author{
Hassan Abdalla ${ }^{1}$, Ali A. Amer ${ }^{\text {Corresp. } 2}$ \\ ${ }^{1}$ College of Technological Innovation, Zayed University, Abu Dhabi, Abu Dhabi, United Arab Emirates \\ 2 Computer Science Department, Taiz University, Taiz, Taiz, Yemen \\ Corresponding Author: Ali A. Amer \\ Email address: aliaaa2004@yahoo.com
}

In Information Retrieval (IR), Data Mining (DM), and Machine Learning (ML), similarity measures have been widely used for text clustering and classification. The similarity measure is the cornerstone upon which the performance of most DM and ML algorithms is completely dependent. Thus, till now, the endeavor in literature for an effective and efficient similarity measure is still immature. Some recently-proposed similarity measures were effective, but have a complex design and suffer some inefficiency. This work, therefore, develops an effective and efficient similarity measure of a simplistic design for text-based applications. The measure developed in this work is driven by Boolean logic algebra basics (BLAB-SM), which aims at effectively reaching the desired accuracy at the fastest run time as compared to the recently developed state-of-the-art measures. Using the term frequency-inverse document frequency (TF-IDF) schema, the K-nearest neighbor (KNN), and the K-means clustering algorithm, a comprehensive evaluation is presented. The evaluation has been experimentally performed for BLAB-SM against seven similarity measures on two most-popular datasets, Reuters-21 and Web-KB. The experimental results illustrate that BLAB-SM is not only more efficient but also significantly more effective than state-of-the-art similarity measures on both classification and clustering tasks. 
1 Boolean Logic Algebra Driven Similarity Measure for

2 Text Based Applications

3

4

5

6

7

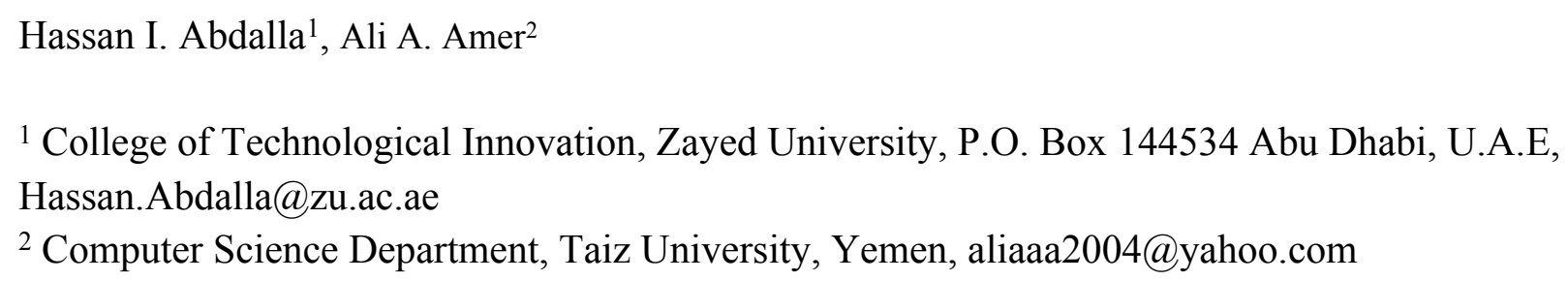

\section{ABSTRACT}

In Information Retrieval (IR), Data Mining (DM), and Machine Learning (ML), similarity measures have been widely used for text clustering and classification. The similarity measure is the cornerstone upon which the performance of most DM and ML algorithms is completely dependent. Thus, till now, the endeavor in literature for an effective and efficient similarity measure is still immature. Some recently-proposed similarity measures were effective, but have a complex design and suffer some inefficiency. This work, therefore, develops an effective and efficient similarity measure of a simplistic design for text-based applications. The measure developed in this work is driven by Boolean logic algebra basics (BLAB-SM), which aims at effectively reaching the desired accuracy at the fastest run time as compared to the recently developed state-of-the-art measures. Using the term frequency-inverse document frequency (TFIDF) schema, the K-nearest neighbor $(\mathrm{KNN})$, and the K-means clustering algorithm, a comprehensive evaluation is presented. The evaluation has been experimentally performed for BLAB-SM against seven similarity measures on two most-popular datasets, Reuters- 21 and Web$\mathrm{KB}$. The experimental results illustrate that BLAB-SM is not only more efficient but also significantly more effective than state-of-the-art similarity measures on both classification and clustering tasks.

\section{INTRODUCTION}

Over the last ten years, Natural Language Processing (NLP) has been subjected to rapid development as new techniques and methods are continuously introduced to satisfy the everdisseminating availability of data. This kind of development is clearly being mostly reflected in the fields of Information Retrieval (IR), Data Mining (DM), and Machine Learning (ML) in which several techniques used the similarity/distance measures for different purposes (Zhang \& Zuo, 
40

41

42

43

44

45

46

47

48

49

50

51

52

53

54

55

56

57

58

59

60

61

62

63

64

65

66

67

68

69

70

71

72

73

74

75

76

77

78

79

80

2019; Gweon, Schonlau \& Steiner, A. 2019; Amer 2020; Teboulle \& Nicholas, 2005; Nguyen \& Amer, 2019).

Several machine learning techniques have recorded a surpassing performance, in the NLP field, to handle the voluminous constantly-piling data and information on the internet. Among these techniques are clustering and classification which are still commonly used in almost all scientific fields including text mining, information retrieval, web search, pattern recognition, and biomedical based text mining (Amer \& Abdalla, 2020; Rachkovskij, 2017; Gweon, Schonlau \& Steiner, B. 2019; Kanungo, Mount, Netanyahu, Piatko, Silverman \& Wu, 2019; Holzinger, Schantl, Schroettner, Seifert \& Verspoor, 2014). For example, in (Holzinger, Schantl, Schroettner, Seifert \& Verspoor, 2014), a detailed survey in biomedical-based text mining and classification was done, while stressing the importance of involving and improving similarity measures for classification tasks. Literature has long been stressing the performance of text clustering and classification which depends mainly on the similarity measures. Essentially, both tasks need highly-effective and maximally-efficient similarity measures to reach the desired rendering. However, finding suitable similarity measures for text clustering and classification is a challenging task. Efficiency and effectiveness are the basic characteristics each similarity measure should enjoy.

Generally speaking, in information retrieval, the documents are drawn as vectors in the vector space model (VSM) (Amer \& Abdalla, 2020). In each document's vector, each cell refers to the value of the relative feature that corresponds to the term presence/absence. In this vector, this value is integer/real number represented by the weighting schema which is commonly falling into one of three cases: (1) term frequency (used in BoW) which indicates the appearance times of the relative term, (2) relative term frequency (TF) which is computed as the ratio between the frequency of term and the net number of appearances of all unique terms in the whole set of documents, (3) TF-IDF which is the most commonly-used schema in information retrieval (Afzali \& Kumar, 2017). Occasionally, documents are broken into a large number of features represented in VSM, resulting in high sparse VSM. In other words, VSM would contain a rather low percentage of non-zero feature values. Consequently, the sparsity along with the dimensionality curse could have a severely negative impact on the performance of classifiers.

To tackle such challenges, in IR literature, a dozen of works have introduced several effective similarity measures for text clustering and classification (Amer \& Abdalla, 2020; Oghbaie \& Mohammadi Zanjireh, 2018; Sohangir ., \& Wang, 2017; Lin, Jiang \& Lee, 2014; Shahmirzadi, Lugowski, \& Younge, 2019; Ke, 2017; White \& Jose, 2004; Lakshmi \& Baskar, 2021; Kotte, Rajavelu \& Rajsingh, 2020; Thompson, Panchev \& Oakes, 2015). However, except for (Amer \& Abdalla, 2020), these studies proposed similarity measures without providing sufficient insights into run-time efficiency. In other words, these studies might introduce effective measures yet timeinefficient. Moreover, these measures, which are shown effective (Amer \& Abdalla, 2020; Oghbaie \& Mohammadi Zanjireh, 2018; Sohangir \& Wang, 2017; Lin, Jiang \& Lee, 2014; Shahmirzadi, Lugowski, \& Younge, 2019; Lakshmi \& Baskar, 2021; Robertson, 2004), suffer the

Peer] Comput. Sci. reviewing PDF | (CS-2020:12:56084:2:0:NEW 10 Jun 2021) 
81 design complexity. Motivated by this, this work comes with the ultimate aim of finding an 82 influential solution to the efficiency as well as the design complexity of those similarity measures. 83 A Boolean logic algebra-driven similarity measure (BLAB-SM) is simply designed with the aim 84 of its being significantly effective and highly efficient. BLAB-SM takes the presence and absence 85 of each term as long as this term exists in either or both documents under consideration, making it 86 highly competitive to give a robust classification. Seven similarity measures are thoroughly 87 examined under diversified conditions. Using TF-IDF representation (Zhao \& Mao, 2018; Joulin, 88 Grave, Bojanowski \& Mikolov, 2017), the K-nearest neighbor (KNN), and the K-means algorithm, 89 this work investigates each similarity measure with varying both the K values of KNN and the

90

91

92

93

94

95

96

97

98

99

100

101

102

103

104

105

106

107

108

109

110

111

112

113

114

115

116

117

118

119

120 number of features of each dataset. Similarly to (Amer \& Abdalla, 2020) that used the BoW model, we have used the TF-IDF model to assess all measures against low dimensional datasets by analyzing performance on $(50,100,200$, and 350 features) and high dimensional datasets ( 3000 , 6000 , and all features of each dataset). The performance of measures was studied profoundly to specify the measures that would yield the desired results in each $\mathrm{K}$ value of KNN and on each number of features. The key contributions, of this work, are drawn as follows:

1. Presenting a new similarity measure whose behavior is driven from the Boolean logic algebra mechanism. It is named Boolean logic algebra-based similarity measure for text clustering and classification (BLAB-SM). Based on a rigorous experimental study, BLAB-SM has been shown a top performer by outperforming all the state-of-the-art measures concerning both effectiveness and efficiency. Indeed, BLAB-SM is one of the fastest measures comparing with all considered measures in this study including cosine, Euclidean, and Manhattan. The experimental results illustrate that BLAB-SM is not only more efficient but also significantly more effective than state-of-the-art similarity measures on both classification and clustering tasks.

2. Drawing a comprehensive and fair evaluation study for BLAB-SM against seven similarity measures. The performance of all similarity measures is benchmarked on web-KB and Reuters-21 datasets. Using TF-IDF, the KNN classifier, and the Kmeans clustering, a comparative study for these measures, regarding the effectiveness and efficiency, is made. Interestingly, this study has experimentally shown that the Jaccard similarity measure is an ineffective measure for TF-IDFbased document matching.

\section{Paper organization}

The related works, including the compared methods in this work, are covered in "RELATED WORK". "THE PROPOSED METHOD" introduces the proposed similarity measure. Experimental settings are concisely presented in "EXPERIMENTAL SETUP". The outcomes of experimental study are drawn in "RESULTS". The most important points out of this study are 
121

122

123

124

125

126

127

128

129

130

131

132

133

134

135

136

137

138

139

140

141

142

143

144

145

146

147

148

149

150

151

152

153

154

155

156

157

158

159

160

161

articulated in "DISCUSSION". Finally, "CONCLUSIONS AND FUTURE WORK" concludes the paper, and presents the avenues of future work.

\section{RELATED WORK}

In IR literature, the vector space model (VSM) has widely been utilized to find the pairwise document similarity using the relative similarity measures. For example, as geometric measures, the Euclidean, Minkowski, Manhattan, and Chebyshev distances are utilized in (VSM) for text classification through finding the distance between each vector pair (Heidarian \& Dinneen, 2016; Cordeiro, Amorim \& Mirkin, 2012). In general, Manhattan distance is mostly more efficient than Euclidean on small datasets, yet it has long been recorded to have less accurate results in most of the text classification tasks particularly with THE sparse data. In our work, Euclidean was seen more efficient when run on web-kb and Reuters, though, That is due to the Manhattan is being contingent upon the rotation of the coordinate system, leading to its being disadvantageous for both document classification and clustering tasks (Kumar, V. , Chhabra \& Kumar, D, 2014). Meanwhile, Jaccard, Ex-Jaccard, Kullback-Leibler divergences (KLD), and Bhattacharya coefficient were all used for the several tasks of ML and IR including text clustering and classification (Amer \& Abdalla, 2020; Tanimoto, 1957; Tata \& Patel, 2007, Oghbaie \& Mohammadi Zanjireh, 2018, François, Wertz, \& Verieysen, 2007; D'hondt, Vertommen, Verhaegen, Cattrysse \& Duflou, 2010; Li, Yao, Fan \& Yu, 2017; Kullback \& Leibler, 1951).

On the other hand, Jaccard is always used to compute the ratio of the number of terms/points included in the document pair (or feature space) to the number of terms included in, at least, either one document/point. However, the Jaccard similarity measure does not utilize the term frequencies in the vector space model, making it an ineffective option for TF-IDF-based matching. Ex-Jaccard, therefore, comes to tackle the limitation of Jaccard when dealing with TF-IDF. However, ExJaccard has long been shown time-inefficient. Both facts have been experimentally corroborated in our study of this paper. In contrast to most similarity measures/ distance metrics, KL divergence is asymmetric. That is, the KLD value from document $\mathrm{d} 1$ to document $\mathrm{d} 2$ does not equal the KLD value from $\mathrm{d} 2$ to $\mathrm{d} 1$. This fact contributes negatively to the performance of KLD when text classification considered. All of these distance/similarity measures are considered among the most efficient methods in IR and ML fields; yet, their performance has not reached the desired effectiveness, or even poor chiefly when the data are sparse or of high dimensions or both combined (Subhashini \& Kumar, 2010; Li \& Han, 2013). On the other hand, Cosine similarity, like Euclidean distance, is one of the most widely-applied similarity measures for text clustering and classification (Amer \& Abdalla, 2020; Arivarasan \& Karthikeyan, 2019; Zhao \& Karypis, 2002). It seeks to find the cosine angle between vectors of each document pair. Cosine has long been shown to be effective and efficient at the same time. However, cosine suffers two limitations: (1) its effectiveness and efficiency have been degraded drastically when applied on highdimensional datasets, or run on datasets of overlapping classes (Amer \& Abdalla, 2020), (2) Cosine is more suitable for text mining when data are a symmetric. Otherwise, Cosine might be either biased or less accurate (Afzali \& Kumar, 2017).

Peer] Comput. Sci. reviewing PDF | (CS-2020:12:56084:2:0:NEW 10 Jun 2021) 
162

163

164

165

166

167

168

169

170

171

172

173

174

175

176

177

178

179

180

181

182

183

184

185

186

187

188

189

190

191

192

193

194

195

196

197

198

199

200

201

To tackle this limitation, therefore, IR and ML literature has still been introducing new similarity measures. Off the most recently-published similarity measures are the set theory-based similarity measure (STB-SM). STB-SM was proposed in (Amer \& Abdalla, 2020) for text classification and clustering, and compared against seven similarity measures in the context of the bag of word model. STB-SM was proven maximally effective and significantly efficient. In (Heidarian \& Dinneen, 2016), a geometric measure was introduced to find the similarity degree between each document pair. Depending on the information theory (D'hondt, Vertommen, Verhaegen, Cattrysse \& Duflou, 2010; Aslam \& Frost, 2003), an Information-Theoretic measure for document Similarity (IT-Sim) was proposed. In the same line, in (Sohangir \& Wang, 2017), a similarity measure, named Improved Sqrt-Cosine similarity (ISC), was developed for text classification, and proven effective. In the same context, the pairwise document similarity measure (PDSM) was proposed in (Oghbaie \& Mohammadi Zanjireh, 2018). PDSM took into account the feature weights and the number of features found in, at least, one document. Another popular similarity measure for text processing, SMTP, was presented in (Lin, Jiang \& Lee, 2014) and shown highly effective when run against the state-of-art similarity measures including cosine, Euclidean and Sim-IT.

Even though the literature has presented a good number of similarity measures, the problem is that these proposed measures were either seen effective like PDSM, SMTP, and ISC; yet, they were time-inefficient. On the other extreme, other measures were seen efficient like Euclidean and Manhattan, yet are not as effective as PDSM, ISC or SMTP. As a compromised solutions, Cosine and STB-SM were described with their being closely effective and reasonably time-efficient. SYBSM was seen much more effective (Amer \& Abdalla, 2020), though. Given the drawn-above limitations of previous similarity measures, our work of this paper endeavors to introduce a novel similarity measure that would tackle these limitations while shrinking the trade-off between efficiency and effectiveness to the greatest extent. The proposed measure behaves based on Boolean logic algebra basis, called BLAB-SM, has been developed, and experimentally shown to outperform all compared state-of-art effectively and efficiently.

\section{The Compared Methods}

In the following, all compared similarity measures and distance metrics are described. Having two documents document 1 and document 2 whose TF-IDF of their "n" terms (w.t) have been saved in both vectors: $\operatorname{doc}_{1}\left(w . t_{11}, w . t_{12}, \ldots, w . t_{1 n}\right)$ and $\operatorname{doc}_{2}\left(w . t_{21}, w . t_{22}, \ldots, w . t_{2 n}\right)$ respectively, the considered seven similarity measures or distance metrics [29-32] are listed as follows;

\section{Euclidean Distance (ED)}

ED computes the distance between each point pair in N-dimensional space. It is define by the following equation:

$$
D_{E u c}(d o c 1, d o c 2)=\sum \sqrt{\left(d o c_{11-} d o c_{12}\right)^{2}+\left(d o c_{21-} d o c_{22}\right)^{2}+\ldots\left(d o c_{n 1-} d o c_{n 2}\right)^{2}}
$$


202

203 204

$$
205 \operatorname{Sim}_{\operatorname{Cos}}(\operatorname{doc} 1, \operatorname{doc} 2)=\frac{\sum_{i=1}^{n} d o c_{i 1} * d o c_{i 2}}{\sqrt{\sum_{i=1}^{n} d o c_{i 1}^{2}} * \sqrt{\sum_{i=1}^{n} d o c_{i 2}^{2}}}
$$

\section{Cosine Measure}

207

The pairwise similarity is found between each document pair using both the dot product as well as

\section{Jaccard Similarity Measure}

It is one of the most widely used similarity measures. However, the problem with Jaccard as we found experimentally is that Jaccard is highly reliant on the common values which are, unlike BoW representation in which Jaccard is working well, barely exist in TF-IDF representation. This reliance factor makes Jaccard a very poor option when used for TF-IDF-based document matching. This measure is defined by next equation.

$$
\operatorname{Sim}_{\text {jaccard }}(\operatorname{doc} 1, \operatorname{doc} 2)=\frac{\operatorname{doc} 1 \cap \operatorname{doc} 2}{\operatorname{doc} 1 \cup \operatorname{doc} 2}
$$

\section{Extended Jaccard}

The extended Jaccard (ex-jaccard, in short) can be considered as the inverse of Jaccard coefficient, and its equation is drawn as follows;

$$
\operatorname{Sim}_{E x-\text { jaccard }}(\operatorname{doc} 1, \operatorname{doc} 2)=\frac{\operatorname{doc} 1 \cdot \operatorname{doc} 2}{|\operatorname{doc} 1|^{2}+|\operatorname{doc} 2|^{2}-(\operatorname{doc} 1 \cdot \operatorname{doc} 2)}
$$

\section{Manhattan}

This distance metric computes the sum of absolute differences between the coordinates of vectors of document pair. It is defined as follows:

$$
\text { Manhattan-distance }(\operatorname{doc} 1, \operatorname{doc} 2)=\sum_{i=1}^{n}\left|\operatorname{doc} 1_{i 1}-\operatorname{doc}_{i 2}\right|
$$

\section{Kullback Leibler Divergence (KLD)}

kld finds the difference between the probability distributions, and is defined as follow;

$\operatorname{Sim}_{K L}(\operatorname{doc} 1, \operatorname{doc} 2)=\sum_{i=1}^{n} P \operatorname{doc} 1\left(\operatorname{doc}_{i 1}\right) * \log \left(\frac{P \operatorname{doc} 1\left(\operatorname{doc}_{i 1}\right)}{P \operatorname{doc} 2\left(\operatorname{doc}_{i 2}\right)}\right)$

where $\operatorname{Pdoc}_{1}\left(\operatorname{doc}_{i 1}\right)$ is the value of $i^{\text {th }}$ feature of $\operatorname{doc}_{1}$ and $\operatorname{Pdoc}_{2}\left(\operatorname{doc}_{i 2}\right)$ is the value of $i^{\text {th }}$ feature of $\operatorname{doc}_{2}$.

\section{SMTP}


233 This similarity measure was presented in (Lin, Jiang \& Lee, 2014) for text clustering and 234 classification. It considers two key scenarios when the feature exists in both documents $\operatorname{doc}_{1}$ and $235 \mathrm{doc}_{2}$, and when the feature is absent in the pair. It was defined as follows;

236

237

238

239

240

241

242

243

244

245

246

247

248

249

250

251

252

253

254

255

256

257

258

259

260

261

262

263

264

265

266

267

$\operatorname{Sim}(\operatorname{doc} 1, \operatorname{doc} 2)=\frac{F(\operatorname{doc} 1, \operatorname{doc} 2)+\lambda}{1+\lambda}$

where

$$
F(\operatorname{doc} 1, \operatorname{doc} 2)=\frac{N_{\text {star }}(\operatorname{doc} 1, \operatorname{doc} 2)}{N_{\text {union }}(\operatorname{doc} 1, \operatorname{doc} 2)}
$$

$$
N_{\text {star }}(\text { doc } 1, \text { doc } 2)=\left\{\begin{array}{l}
0.5\left(1+\exp ^{-\left(\frac{d o c_{i 1}{ }^{d o c_{i 2}}}{v a r}\right)^{2}}\right) \text { if doci1.doci } 2>0 \\
0 \text { if } \text { doc }_{i 1}=0, d o c_{i 2}=0 \\
-\lambda \text { Otherwise }
\end{array}\right.
$$

And

$$
N_{\text {union }}(\text { doc } 1, \text { doc } 2)=\left\{\begin{array}{l}
0, \text { if doci } 1=0, \text { doci } 2=0 \\
1, \text { Otherwise }
\end{array}\right.
$$

Where $\lambda$ is a constant that was fixed at values of (1) and (0.01), while conducting our experiments, for classification and clustering respectively. The variable var indicates the standard distribution of all non-zero features. The values of (1) and (0.01) were not randomly chosen for $\lambda$. They were selected based on the setting asserted and used in (Lin, Jiang \& Lee, 2014) as the optimal option to compare SMTP with other measures for both classification and clustering.

\section{THE PROPOSED METHOD}

\section{Motivation}

As mentioned earlier in the drawn-above sections, the text classification and clustering is tricky task chiefly when big data is set to be handled. Furthermore, even though the literature is full of effective similarity measures that have a good performance, the necessity for efficient and effective similarity measures is still incomplete. While there have been some efficient measures like Euclidean and Manhattan, these measures have been shown of poor performance when applied on texts of middle, big, or voluminous-sized datasets. On the other hand, there have been efficient and effective measures like cosine; however, cosine measure does not reach the desired performance. Therefore, several works in literature have been presenting new effective measures like PDSM, ISC, SMTP, and STB-SM. While PDSM was seen as a rather time-inefficient measure (Amer \& Abdalla, 2020), SMTP has been seen time-inefficient as well in our work. On the other hand, these measures were seen much more effective than cosine; Yet not as efficient as cosine. The need to address the trade-off between efficiency and effectiveness motivates us mainly to enrich IR and ML with a new efficient and effective similarity measure (BLAB-SM). The newlyproposed measure has been proposed to shrink the already-mentioned trade-off. Experimentally, 
268

269

270

271

272

273

274

275

276

277

278

279

280

281

282

283

284

285

286

287

288

289

290

291

292

293

294

295

296

297

298

299

300

301

302

303

304

305

306

307

308

309

BLAB-SM is seen as effective as SMTP, and more efficient than cosine. It is also seen as efficient as Euclidean and Manhattan in most cases which is the ultimate objective of this work. Concisely, BLAB-SM comes to effectively fill the gap of efficiency problem from which some effective measures like SMTP suffers, while maintaining, if not outperforming in some cases, the effectiveness reached by SMTP.

\section{BLAB-SM Similarity Measure}

Three basic definitions for the Boolean logic algebra, which inspired us to propose the measure, are briefly highlighted before presenting our proposed measure, BLAB-SM. In doing so, BLABSM's explanation and analysis would be completely understood.

\section{The Logical Gates}

Strictly speaking, the digital systems are constructively defined by using the logic gates. In general, the basic gates are the AND, OR, NOT gates. The basic operations are described below along with their truth tables as follows;

Figure 1 AND Gate Table 1 A AND B Truth Table

The AND gate is an electronic circuit that yields an output (1) only and only if all its inputs (A and B, in Figure 1, Table 1) are (1). This symbol $(\Lambda)$ is used to show the AND operation, i.e. A $\wedge$ B. AND gate in our measure comprises only and only the shared features.

Figure 2 OR Gate Table 2 A OR B Truth Table

Like AND gate, the OR gate is also an electronic circuit. However, the OR gate yields an output (1) if and only if one or more of its inputs (A and B, in Figure 2, Table 2) are (1). This symbol (V) is used to show the OR operation, i.e. A $\vee \mathrm{B}$. OR gate in our measure concerns both the shared and non-shared features at the same time.

Figure 3 EXOR Gate Table 3 A EXOR B Truth Table

The 'Exclusive-OR' gate is a circuit that yields an output (1) if either one, but not both, of its two inputs are (1). The symbol with an encircled plus sign $(\oplus)$ is utilized to signal the Ex-OR operation, i.e. $A \oplus B$ (see $A$ and $B$ in Figure 3 and Table 3). EXOR gate in our measure concerns only and only the non-shared features so as to non-shared features are given a chance to contribute in calculating similarity degree.

\section{The Boolean Logic Algebra Based Similarity Measure (BLAB-SM)}

Based on the already drawn concepts, the Boolean algebra based similarity measure (BLAB-SM) is defined as follows;

$$
B L A B-S M(\operatorname{doc} 1, \operatorname{doc} 2)=\alpha \times X+\beta \times Y
$$


311 Both $\alpha$ and $\beta$ are parameters that have experimentally been tuned, as drawn in the results section, 312 just to find their best values to detect the highest possible similarity. Although X is concerned with 313 finding similarities between all non-shared features, it finds similarity between all shared features 314 implicitly as well. It finds similarities based on logical sensing of the differences between both 315 documents under consideration. All documents are logically treated and processed the same way 316 logical gates work. On the other hand, Y emphasizes similarity through finding likeness between 317 documents across all shared features only. Assuming having two documents which are document 1 318 and document 2 which are represented by vectors $\operatorname{doc}_{1}$ and $\operatorname{doc}_{2}$ in the vector space model using 319 TF-IDF representation. Using $\operatorname{doc}_{1}$ and $\operatorname{doc}_{2}$ with " $n$ " terms, $\mathrm{X}$ and $\mathrm{Y}$ are defined as follows;

$$
\begin{aligned}
& X=\left(\begin{array}{r}
\sum_{I=1}^{n}\left(d o c_{i 1} \oplus d o c_{i 2}\right) \\
\sum_{I=1}^{n}\left(d o c_{i 1} \vee d o c_{i 2}\right)
\end{array}\right) \\
& Y=\left(\frac{2 \times\left(\sum_{I=1}^{n}\left(d o c_{i 1} \wedge d o c_{i 2}\right)\right)}{\left(\sum_{I=1}^{n} d o c_{i 1}\right)+\left(\sum_{I=1}^{n} d o c_{i 2}\right)}\right)
\end{aligned}
$$

322

323

324

325

326

327 328 329

330

331

332

333

334 335

Meanwhile, $\operatorname{doc}_{1}$ or $\operatorname{doc}_{2}=1$, if $\operatorname{doc}_{1}$ or $\operatorname{doc}_{2} \geq 1$; Otherwise, 0 .

\section{BLAB-SM Analysis}

In this sub-section, we concisely, simply and informatively analyze the cases of the proposed measure. Assuming that $\mathrm{X}$ consists of $X_{1}$ and $X_{2}$, and $\mathrm{Y}$ consists of $Y_{1}, Y_{2}$, and $Y_{3}$. Accordingly, the $\mathrm{X}$ and $\mathrm{Y}$ equations are re-drawn as follows;

$$
X=\left(1-\frac{X_{1}}{X_{2}}\right)
$$

where:

$$
X_{1}=\sum_{I=1}^{n}\left(d o c_{i 1} \oplus d o c_{i 2}\right)
$$

$$
X_{2}=\sum_{I=1}^{n}\left(d o c_{i 1} \vee d o c_{i 2}\right)
$$

Similarly:

$$
Y=\left(\frac{2 \times Y 1}{Y 2+Y 3}\right)
$$


336

337

338

339

340

341

342

343

344

345

346

347

348

349

350

351

352

353

354

355

356

357

358

359

360

361

362

363

364

365

366

367

368

369

370

371

372

where:

$$
\begin{gathered}
Y_{1}=\sum_{I=1}^{n}\left(\operatorname{doc}_{i 1} \wedge d o c_{i 2}\right), \\
Y_{2}=\sum_{I=1}^{n} d o c_{i 1} \text {, and } Y_{3}=\sum_{I=1}^{n} d o c_{i 2}
\end{gathered}
$$

The perfect dissimilarity case:

This case happens when:

$$
X_{1}=X_{2} \Rightarrow \mathrm{X}=\text { zero, because } \mathrm{X} \text { would equal }(1-1=0)
$$

$$
\begin{aligned}
& \because X_{1}=X_{2} \Rightarrow \quad Y_{1}=\text { zero }\left(i t \text { is here AND gate), } X_{1}=1 \text { since } \frac{X_{1}}{X_{2}}=1\right. \\
& \Rightarrow Y=\frac{2 \times \text { zero }}{Y_{2}+Y_{3}}=\text { zero, regardless of } Y 2 \text { and } Y 3 \text { values }
\end{aligned}
$$$$
\Rightarrow \mathrm{BLAB}-\mathrm{SM}(\operatorname{doc} 1, \operatorname{doc} 2)=\alpha \times X+\beta \times Y=\alpha \times \text { zero }+\beta \times \text { zero }=\text { zero }
$$

Example (perfect dissimilarity case): assuming we have doc1 $(3,0,1)$ and doc $2(0,2,0)$. By the applying the perfect dissimilarity scenario, we find that BLAB-SM=zero, for both documents (1, $0,1)$ and $(0,1,0)$, which is logically true since there is no shared feature exist.

The average similarity:

$$
\begin{aligned}
X_{2}>X_{1}>\text { zero } \Rightarrow Y_{1}>\text { zero } & \Rightarrow 1>\mathrm{X}>\text { zero, and } 1>\mathrm{Y}>\text { zero } \\
& \Rightarrow 1>\mathrm{BLAB}-\mathrm{SM}(\operatorname{doc} 1, \text { doc } 2)>\text { zero }
\end{aligned}
$$

Where (1) is the upper bound and (0) is the lower bound.

Example (Average similarity): assuming we have doc $1(3,1,1)$ and doc $2(6,2,0)$. By applying the average case scenario on both documents $(1,1,1)$ and $(1,1,0)$, BLAB-SM would have a value of roughly $(0.73)$ which is bigger than zero and less than 1 . It is worth indicating that 0.73 is logically reasonable than the cosine value which would reach 0.95 . In fact, this is one novelty of our measure as similarity has never been exaggerated like what is done with the most state-ofart measure. Our measure allows non-zero non-shared features to have an implicit contribution to the similarity computation. Therefore, BLAB-SM takes the presence and absence of all features into consideration effectively.

The perfect similarity case:

$$
X_{1}=\text { zero } \Rightarrow \mathrm{X}=1-\frac{\text { zero }}{X_{2}},
$$

$$
\mathrm{X}=1 \text {, regardless of the value of } X_{2}
$$

$$
\begin{aligned}
& \because X_{1}=\text { zero } \Rightarrow Y_{1}=\overline{X_{1}}, \\
& \Rightarrow Y_{1}=1 \text {, since } Y_{1}=\text { complement }\left(X_{1}\right) \Rightarrow \mathrm{Y}=\frac{2 \times 1}{Y_{2}+Y_{3}}
\end{aligned}
$$$$
\because Y_{1}=1 \Rightarrow \text { Length }(\text { doc } 1)=\text { Length }(\text { doc } 2) \Rightarrow Y_{2}=Y_{3}
$$

$$
\Rightarrow Y=\frac{2}{2 x Y_{2}\left(\text { or } 2 x Y_{3}\right)}=\frac{2}{2}=1
$$


373 Example (perfect similarity Case): assuming we have doc1 $(3,3,3)$ and doc2 $(3,3,3)$, or doc1 $374(1,1,1)$ and doc2 $(1,1,1)$. By applying the best case scenario, we find that BLAB-SM=1 which 375 is logically true as both documents are equal and equivalent.

376

377

\section{Similarity Measure Characteristics}

378

379

380

381

382

383

384

385

386

387

388

389

390

391

392

393

394

395

396

397

398

399

400

401

402

403

404

405

406

407

408

409

410

411

Six characteristics should be defined on each similarity measure so this measure could be classified as good measure (Haroutunian 2011; Amigó, Giner, Gonzalo, \& Verdejo, 2020). These characteristics are given as follows;

Characteristic 1: The presence or non-presence of the targeted feature is more important than the difference between the values connected with the present feature.

Assuming we have $\operatorname{doc}_{1}(3,1,1)$ and $\operatorname{doc}_{2}(6,2,0)$. Then, the binary vectors, $(1,1,1)$ and $(1,1$, 0 ), of both documents are more important than the integer values linked to the values of features themselves in both documents. Let us take feature $3\left(f_{3}\right)$, we can say that feature 3 has no link with doc $_{2}$ while it has a link with doc $_{1}$. In such case, both documents are dissimilar with respect to feature 3 . So, we can conclude that feature 3 has more weight in deciding similarity between doc $_{1}$ and $\operatorname{doc}_{2}$ than $\mathrm{f}_{1}$ and $\mathrm{f}_{2}$ which are exist in both documents. In fact, BLAM-SM focus in this issue when similarity is being calculated as behavior of BLAM-SM goes in the same way the logic of gates mechanism works.

Characteristic 2: The similarity degree should increase when the difference between the nonzero features values decrease. For example, having feature 1 and feature 2 as two features $\left(f_{1}\right.$ and $\mathrm{f}_{2}$ ) of $\operatorname{doc}_{1}$ and $\operatorname{doc}_{2}$, similarity $\left(\operatorname{doc}_{1}, \operatorname{doc}_{2}\right)$ while $\mathrm{f}_{1}=10$ and $\mathrm{f}_{2}=5$ is higher than the similarity between $\mathrm{f}_{1}=18$ and $\mathrm{f}_{2}=4$.

Characteristic 3: The similarity degree should be increased when the number of present features grows. For example, having three vectors of three documents; $\operatorname{doc}_{1}(1,1,0), \operatorname{doc}_{2}(1,1,1), \operatorname{doc}_{3}$ $(1,0,0)$, the similarity $\left(\operatorname{doc}_{1}, \mathrm{doc}_{2}\right)$ is far higher than similarity $\left(\mathrm{doc}_{1}, \mathrm{doc}_{3}\right)$ due to the difference in the number of present or non-present features between all documents.

Characteristic 4: The document Pairs are highly different to each other if there have not had almost equivalent zero-valued features versus non-zero-valued features. For example, having two vectors of two documents $\operatorname{doc}_{1}\left(f_{1}, f_{2}\right)=(1,0)$ and $\operatorname{doc}_{2}\left(f_{3}, f_{4}\right)=(1,1)$, doc $1 . f_{2}$ and $\operatorname{doc}_{2} \cdot f_{4}$ are the main reason for maximizing the difference between both documents as $\mathrm{f}_{2} * \mathrm{f}_{4}=0$, and, $\mathrm{f}_{2}+\mathrm{f}_{4}>0$.

Characteristic 5: the symmetric properties should be met by each similarity measure. For example, the similarity degree between $\operatorname{doc}_{1}(0,1,1,0)$ and $\operatorname{doc}_{2}(1,1,1,1)$ must be the same when $\operatorname{doc}_{2}(1,1,1,1)$ and $\operatorname{doc}_{1}(0,1,1,0)$ are addressed.

412 
413 Characteristic 6: The value of distribution should contribute to the similarity degree between 414 each document pair.

415

416

\section{EXPERIMENTAL SETUP}

417 In the following, the experimental settings, algorithms, machine and data sets' descriptions, 418 performance evaluation for both classification and clustering tasks are all elaborated.

419

420

421

422

423

424

425

426

427

428

429

430

431

432

433

434

435

436

437

438

439

440

441

442

443

444

445

446

447

448

449

450

For text clustering and classification applications, the feature extraction/selection and the preprocessing are crucial stages. First of all, text pre-processing was done to make the text processable. Initially, the text was switched from upper case to lower case, and numbers, punctuations, stop words (common words) were removed. The extra spaces and symbols (like \$, \%) were also eliminated. For word stemming and text representation, python 3 was used to run the preprocessing using Nltk (Natural language toolkit) library. The ntlk word tokenizer was used for tokenization, and the Lemmatizing was done using the ntlk stem WordNetLemmatizer. Finally, stopword removal was done using the ntlk stopwords. Then, the vector space model (VSM) was utilized to represent features with TF-IDF representation (Robertson 2004). The TF-IDF is the multiplication of term frequency (TF) by the inverse document frequency (IDF), and is defined as follows;

$$
\begin{aligned}
& T F-I D F_{(t, d)}=t f(t, d o c) * \log \left(\frac{N}{d f+1}\right) \\
& \text { where, } t f(t, d)=\frac{\text { count of term } t \text { in document doc }}{\text { number of words in document doc }}
\end{aligned}
$$

Where $\mathrm{df}$ is the occurrence of $\mathrm{t}$ in all considered documents, and $\mathrm{N}$ is the total number of documents.

\section{KNN Classifier}

In general, $\mathrm{KNN}$ examines each test point based on its " $K$ " closest "neighboring" points which are sorted and assumed to be the most similar points. Then, the test point is classified based on the voting technique. It takes the class label of the majority vote among all $K$ neighboring training points in the feature space. The neighborhood is basically determined based on the used distance/similarity measure. Let us assume that $d_{i}$ is the training set, and $d_{i}^{*}$ is the testing set, $c$ is the true class of training set, and $c^{*}$ is the predicted class for the testing set $\left(c, c^{*}=\ldots, m\right)$ where $\mathrm{m}$ is the number of classes. In the training classification process, only the true class $\mathrm{c}$ of each training set is used, and during testing, class $\mathrm{c}^{*}$ is predicted for each testing set. On the other hand, when using the one-nearest neighbor rule, the predicted class of the testing set $d_{i}{ }^{*}$ is set equal to the true class $\mathrm{c}$ of its nearest neighbor, where $\mathrm{w}_{\mathrm{i}}$ is the nearest neighbor to $\mathrm{d}^{*}$ at the distance:

$$
d\left(w_{i}, d_{i}^{*}\right)=\min _{j}\left\{d\left(w_{j}, d_{i}^{*}\right)\right\}
$$


451 For KNN, the predicted test sample class $d_{i}^{*}$ is assigned to the most frequent true class among 452 the K nearest training sets.

453

454 The experimental design flow chart, provided in Figure 4, shows briefly how the KNN classifier 455

456

457 was used to perform classification task successfully.

458

Figure 4. The experimental Design Flow Chart

459

460

\section{Machine Description}

461 This work has been written in python language, and run on Processor Intel Core i5-3320M (2.6 GHz), RAM 4GB with OS Windows 7 (64 bit).

463

464

465

466

467

\section{Datasets Description}

468

Reuters-R8 (Table 4): it consists of 7674 documents with eight classes, and has 18308 features after it has been pre-processed.

470 Web-kb (Table 5): it consists of 4199 documents with four classes, and has 33,025 features after it has been processed. It is composed of web pages of computer science from universities:

472 Cornell, Texas, Washington, and Wisconsin.

473

474 Both Tables (4) and (5) holds the description of both datasets used in this work. The data in each 475 datasets was split into training and testing in ratio $2: 1(67 \%: 33 \%)$. It is worth indicating that these datasets are publicly available ${ }^{1}$.

484

485

Table 4 Reuters-R8 dataset -8 Classes

Table 5 Web-KB dataset -4 Classes

\section{Classification Evaluation Criterion}

\section{Accuracy}

It checks the sample total that are unmistakably classified out of the whole collection.

$$
\text { ACC }=\frac{\text { True Positive }+ \text { True Negative }}{\text { True Positive }+ \text { True Negative }+ \text { False Positive }+ \text { False Negative }}(11)
$$

\section{Precision}

486 It gives the whole number of items unmistakably identified as positive out of the whole items 487 defined as positive.

$488 \quad$ PRE $=\frac{\text { True Positive }}{\text { True Positive + False Positive }}$

\footnotetext{
${ }^{1}$ https://gith ub.com/aliamer/Boolean-Logic-Algebra-Driven-Similarity-Measure-for-Text-BasedApplications/blob/main/Reuters\%20\%2B\%20WebKB\%20datasets.rar
} 
489

490

\section{Recall}

491 It gives the whole number of items unmistakably identified as positive out of the actual positive

$492 \mathrm{REC}=\frac{\text { True Positive }}{\text { True Positive }+ \text { False Negative }}$

493

494 F-Method

495 It is a harmonic mean of precision and recall.

$496 \quad$ F Score $=2 * \frac{\text { Precision } * \text { Recall }}{\text { Precision }+ \text { Recal }}$

497 G-Method

498 It is used as a geometric mean of both precision and recall.

499

$\mathrm{GM}=\frac{\text { True Positive }}{\sqrt{(\text { True Positive }+ \text { False Positive }) *(\text { True Positive }+ \text { False Negative })}}(15)$

500

501

502

\section{Average Mean Precision (AMP)}

503 It is the average of the averaged precision of all classes.

$504 A M P=\sum_{n}\left(R_{n}-R_{n-1}\right) P_{n}$

505 Where $P_{n}$ and $R_{n}$ are the precision and recall at the $\mathrm{n}^{\text {th }}$ threshold.

506

507

508

509

\section{Clustering Evaluation Criterion}

510 To check the purity of each cluster as it judges the coherence of a cluster.

511

$$
\text { Purity }=\frac{1}{N} \sum_{i=1}^{k} \max _{j}\left|c_{i} \cap t_{j}\right|
$$

512 Where $\mathrm{N}=$ number of data points, $\mathrm{k}=$ number of clusters, $\mathrm{c}_{\mathrm{i}}$ is a cluster in $\mathrm{C}$, and $\mathrm{t}_{\mathrm{j}}$ is the

513 classification which has the max count for cluster $\mathrm{c}_{\mathrm{i}}$

514

515 Entropy

516 It is used to measure the extent to which a cluster contain single class and not multiple classes.

517

$$
E N=\sum_{i=1}^{c} c i * \log (c i)
$$

518

519 RESULTS

520 Before endorsing the best values of parameters $\alpha$ and $\beta$, each parameter was thoroughly and

521 carefully tested while its value varied from (0.1) to (0.9) with an increment of (0.1) each time.

522 Varying values from 0.1 to 0.9 for both parameters was to test BLAB-SM on all different

523 scenarios so the desired effect would not be missed. Based on the results of the thoroughly- 
524 performed experiments on several versions of BLAB-SM, we approved values $(0.5 ; 0.5)$ for $\alpha$

525

526

527

528

529

530

531

532

533

534

535

536

537

538

539

540

541

542

543

544

545

546

547

548

549

550

551

552

553

554

555

556

557

558

559

560

561

562

563

564

565

and $\beta$ respectively when running BLAB-SM for both classification and clustering tasks.

\section{Classification Results}

Fixing $\alpha$ and $\beta$ on values $(0.5 ; 0.5)$, this work examines all similarity measures thoroughly using KNN classifier and K-means clustering algorithm. The $\mathrm{K}$ value in $\mathrm{KNN}$ was changed in each run from (1) to (120) as given in the appendix. Furthermore, the number of features was falling in one of these values $(10,50,100,200,350,3000,6000, \mathrm{NF})$ where NF is the whole number of features. After that, the results are averaged for each measure on all $\mathrm{K}$ values as given in Tables (6-14). On the other hand, albeit it has long been proven effective when dealing with the BoW model, the Jaccard measure has been excluded due to its being proven ineffective option when dealing with TF-IDF representation (see the appendix). That is because of the fact that Jaccard is heavily based on the common values between each considered pair. However, the common values with TF-IDF is far less than it is with BoW. This fact justifies the poor behavior of Jaccard when dealing with TF-IDF-based document matching. The bolded values in the next Tables signify the best values. For readability, Accuracy, Precision, Recall, F-Measure, GMeasure, and Average Mean Precision are represented by ACC, PRE, REC, FM, GM, and AMP respectively.

Table 6. Performance Evaluation, $\mathrm{NF}=10$ - Averaged Results

In Table 6, for Reuters dataset, the BLAB-SM similarity measure, Manhattan followed by KLD achieved the highest accuracy. Ex-jaccard, although it was not among the best performers but it still outperformed Euclidean, SMTP, and cosine respectively with regard to accuracy. On the other hand, KLD followed by BLAB-SM and Manhattan had the best FM and AMP with BLAB is better with FM. However, with regard to the Web-KB dataset, Manhattan, followed by KLD and Euclidean, met the highest accuracy. For FM and AMP criterions, Manhattan followed by Euclidean and cosine, outweighed all other measures. Thus, the best measures were BLAB-SM, Manhattan, and KLD on Reuters, and Manhattan followed by KLD and Euclidean on Web-KB.

Table 7. Performance Evaluation, $\mathrm{NF}=50-$ Averaged Results

Table 8. Performance Evaluation, NF=100- Averaged Results

In Tables 7-8, it is obvious that, for Reuters dataset, BLAB-SM followed by SMTP and cosine, obtained the highest ACC. However, with FM and AMP, Manhattan, Euclidean, cosine and SMTP obtained the best values. On Web-KB dataset, SMTP, followed by BLAB-SM, and cosine, achieved the highest ACC, FM and AMP respectively. Thus, the top performer measures were SMTP, BLAB-SM, and cosine. From Table 9, on Reuters and Web-KB datasets, BLABSM, followed by SMTP and cosine, obtained the highest ACC, FM, and AMP with cosine being superior over BLAB-SM and SMTP in terms of FM and AMP. Thus, the top performers were BLAB-SM, SMTP, and cosine.

Peer] Comput. Sci. reviewing PDF | (CS-2020:12:56084:2:0:NEW 10 Jun 2021) 
566

567

568

569

570

571

572

573

574

575

576

577

578

579

580

581

582

583

584

585

586

587

588

589

590

591

592

593

594

595

596

597

598

599

600

601

602

603

604

605

606

607

608

609

Table 9. Performance Evaluation, $\mathrm{NF}=200-$ Averaged Results

In Table 10, on Reuters dataset, Cosine, followed by BLAB-SM and SMTP, obtained the highest ACC. Interestingly, Cosine had been superior to BLAB-SM and SMTP with FM and AMP. However, on Web-KB dataset, like Table 9, BLAB-SM, SMTP followed by cosine obtained the highest ACC, FM, and AMP respectively. Thus, the top performers were BLAB-SM, SMTP, and cosine.

Table 10 Performance Evaluation, $\mathrm{NF}=350-$ Averaged Results

From Table 11, on Reuters dataset, SMTP, followed by BLAB-SM and cosine, obtained the highest ACC, FM, and AMP with cosine being superior over SMTP and BLAB-SM in terms of FM and AMP. However, on Web-KB dataset, BLAB-SM, followed by SMTP and cosine, obtained the highest ACC, FM and AMP respectively. Thus, the top performers were BLABSM, SMTP and cosine.

Table 11 Performance Evaluation, $\mathrm{NF}=3000-$ Averaged Results

In Tables 12-13, on Reuters dataset, SMTP, followed by BLAB-SM and Ex-jaccard, obtained the highest ACC. Cosine, Euclidean, SMTP, and BLAB-SM were the best on FM and AMP. However, on Web-KB dataset, BLAB-SM, followed by SMTP and cosine, obtained the highest ACC, FM, and AMP with cosine being superior over BLAB-SM and SMTP in terms of FM only. Thus, the top performers were BLAB-SM, SMTP, Ex-Jaccard and cosine.

Table 12. Performance Evaluation, $\mathrm{NF}=6000-$ Averaged Results

Table 13. Performance Evaluation, $\mathrm{NF}=$ the whole Number of Features

Finally, and most importantly in the averaged results case, in Table 14, BLAB-SM and SMTP followed by Cosine were the best measures on both datasets with BLAB-SM taking the lead over SMTP, and SMTP taking the lead over cosine. Surprisingly, cosine/Reuters was the top in terms of FM and AMP.

Table 14. Performance Evaluation - the Averaged of Averaged Results

\section{Clustering Results}

We fixed $\mathrm{K}$ on 5,10 , and the number of actual classes of each dataset. The K-means was conditioned to stop after (50) iterations, or the stability has been reached after two successive cycles. Centroids were randomly picked in each iteration. The best measures based on Table 15, BLAB-SM went side by side with Euclidean followed by SMTP and KLD. The bolded values in Tables (15-16) signify the best values.

Table 15. Purity

On the other hand, based on results of Table 16, Manhattan, BLAB-SM, SMTP followed By Cosine have been the best measures.

Table 16. Entropy

Peer] Comput. Sci. reviewing PDF | (CS-2020:12:56084:2:0:NEW 10 Jun 2021) 


\section{DISCUSSION}

612 In this section, we have investigated two key points: (1) the stability of each similarity measure

613

614

615

616

617

618

619

620

621

622

623

624

625

626

627

628

629

630

631

632

633

634

635

636

637

638

639

640

641

642

643

644

645

646

647

648

649

650

over each dataset, (2) which features number each similarity measure has been the best.

\section{Measures Stability}

Recalling the results of Tables 6-14, Table 17 holds the most stable measures. In Table 17, R and W refer to both Reuters and Web-KB respectively.

Table 17. Measure Stability Status

Table 17 shows that SMTP, BLAB-SM and cosine as the most stable measures with 40, 39, and 39 points respectively. It gives one plus $(++1)$ for each measure when a measure has been superior in terms of any criterion (ACC, FM, and AMP). On Reuters, the competition was held between BLAB-SM, SMTP, cosine, and Ex-Jaccard. Moreover, it can be concluded, from Table 17, that BLAB-SM, SMTP, cosine can be used effectively for both low, middle and high dimensional datasets on each NF. Euclidean and Manhattan also performed well on low dimensional datasets (NF in [10-200] features). EX-Jaccard was observed to behave well on the middle and high dimensional datasets (NF in [350-N] features).

\section{Performance Analysis}

In this section, using the accuracy, f-measure, and average mean precision, we analyze the measure's performance.

\section{Reuters}

Figure 5 shows BLAB-SM, SMTP, Ex-Jaccard, and Cosine achieved the most stable performance. The Cosine and ex-Jaccard showed a punctuated accuracy as cosine was superior when NF was in the range [10-3000]. Ex-Jaccard had the lead as NF grew, though. When $\mathrm{NF}=6000$, competition restricted among Cosine, Ex-jaccard, SMTP, and BLAB-SM with SMTP, BLAB-SM, and Ex-Jaccard being the best. However, as NF exceeded 6000 features, the competition was held between SMTP, BLAB-SM, and Cosine with SMTP and BLAB-SM being the top performers. On the other hand, Manhattan, Euclidean, and kullback Leibler had the worst performance chiefly as NF grew. Albeit that their performance is good when the number of features (NF) were between 10 and 350, performance deteriorated steadily as NF surpasses 350 .

Figure 5 Accuracy over all Measures on all NF values- Average results (K=1-120; +2) - Reuters

In general, based on Figures 6 and 7, it can be concluded that Cosine, BLAB-SM, and SMTP had an almost close performance with slight superiority given to Cosine. Euclidean was shown a competitor with cosine when all features were considered, though. As expected, KLD, Manhattan, Euclidean had poor performance compared to BLAB-SM, SMTP, and Cosine. The Ex-jaccard had a competitive performance on Reuters, though. 
651

652

653

654

655

656

657

658

659

660

661

662

663

664

665

666

667

668

669

670

671

672

673

674

675

676

677

678

679

680

681

682

683

684

685

686

687

688

689

690

691

692

Figure 6 F-measure over all Measures on all NF values- Average results $(\mathrm{K}=1-120 ;+2)$ - Reuters

Figure 7 AMP over all Measures on all NF values- Average results $(\mathrm{K}=1-120 ;+2)-$ Reuters

\section{Web-Kb}

Figures (8-10) illustrate the map of the criterion movements (results were averaged) for all measures over several NF values. On one hand, in Figure 8, Cosine had been the middle ground between the first group of measures (KLD, Manhattan, Euclidean, and Ex-Jaccard) and those with the best performance (BLAB-SM and SMTP). BLAB-SM and SMTP had been involved in fierce competition with SMTP being superior as NF was in the range [10-100]. However, BLABSM had shown higher superiority as NF grew and surpassed 200 features. On the other hand, Figure 8 reveals that Manhattan and kullback Leibler performed poorly as NF grew.

Surprisingly, Ex-Jaccard had poor performance as NF was in the range [10-200]; yet, as NF grew, its performance grew and was competitive with Euclidean and cosine.

Figures 9 and 10, on the other hand, show almost the same conclusion deduced from figure 7, with BLAB-SM being better than SMTP in terms of FM and AMP. In general, like Reuters, both BLAB-SM and SMTP had a close performance trend with significant superiority given to BLAB-SM regarding ACC and AMP. Interestingly, cosine was highly competitive with BLABSM and SMTP in terms of FM and AMP. Euclidean was an equivalent to cosine when all features were considered, though. Finally, KLD, Manhattan, Euclidean, and ex-Jaccard had lower performance on Web-KB compared with BLAB-SM, SMTP, and Cosine. Similarly to Reuters, Euclidean was superior to Ex-Jaccard when NF was in the range [10-350]. However, Ex-Jaccard had the lead when NF grew except for one case.

Figure 8. Accuracy over all Measures on all NF values- Average results $(\mathrm{K}=1-120 ;+2)-\mathrm{Web}-\mathrm{KB}$

Figure 9 F-measure over all Measures on all NF values- Average results $(\mathrm{K}=1-120 ;+2)-\mathrm{Web}-\mathrm{KB}$

Figure 10 AMP over all Measures on all NF values- Average results $(\mathrm{K}=1-120 ;+2)-\mathrm{Web}-\mathrm{KB}$

Last but not least, Figures 11-12 showcase the averaged performance of all measures on both datasets. Both figures stress that BLAB and SMTP have an almost equal performance trend as the top performers.

Figure 11. Performance of all Measures on both datasets - Reuters and Web-KB

Figure 12. Performance of all Measures on both datasets - Reuters and Web-KB

Peer] Comput. Sci. reviewing PDF | (CS-2020:12:56084:2:0:NEW 10 Jun 2021) 
693 Finally, to provide the statistical evidence for the robustness of BLAB-SM's performance, the

694

695

696

697

698

699

700

701

702

703

704

705

706

707

708

709

710

711

712

713

714

715

716

717

718

719

720

721

722

723

724

725

726

727

728

729

730

731

732

733

734

735

736 results of BLAB-SM against its rival measures have been verified on both datasets using the standard test of the non-parametric Wilcoxon test and the paired t-test. The statistical details for BLAB-SM, against all seven similarity measures, have been made based on the Accuracy "ACC" metric on both datasets, and the results are drawn in Tables 18-23. Results show that BLAB-SM is highly effective on Web-KB, and competitively effective with SMTP and ExJaccard on Reuters. The statistical analysis is made by setting the standard value of the significance level at 0.05 (95\%), and results are analyzed regarding the MAE, MSE, Std Error, zscore, $p$-value, and t-score. The degrees of freedom (DF) for the paired t-test have been assigned the value of 59.0 as we have $60 \mathrm{~K}$ values $(1-120,+2)$ for the KNN classifier (see Appendix). The negative sign of $z$-score and t-score implies the effectiveness and superiority of BLAB-SM comparing with its rival measures. In other words, both z-score and t-score indicate the significant difference between the BLAB-SM and its rivals.

Table 18. Reuters - Statistical Significance of the experimental results of BLAB-SM against all measures

Table 19. Reuters -Statistical significance with non-parametric Wilcoxon for BLAB-SM against all measures

Table 20. Reuters -Statistical significance with paired t-test (DF is degrees of freedom) for BLAB-SM against all measures

Table 21. Web-KB - Significance Test of BLAB-SM against all measures

Table 22. Web-KB - Statistical significance with non-parametric Wilcoxon for BLAB-SM against all measures

Table 23. Web-KB - Statistical significance with paired t-test (DF stands for degrees of freedom)

Furthermore, for both datasets, the p-value of BLAB-SM is less than the value of the significance level (0.05). This is strong evidence for the performance robustness of BLAB-SM comparing with its rival measures. On one hand, based on the statistical results given in Tables 18-23, the negative sign of both z-score and t-score shows that the BLAB-SM measure is the top performer comparing with its rival measures. Moreover, z-score values of the BALB-SM measure are maximally significant in all cases as its p-values are less than $(0.05)$ which is the level of significance. On the other hand, on Reuters only, in terms of t-score, all the results are significant except of SMTP and Ex-jaccard. Nevertheless, this slight insignificance of BLABSM could be compensated by the maximally-significant run time of BLAB-SM against both SMTP and Ex-Jaccard on Reuters.

\section{Clustering Analysis}

Using the results of Tables 15-16, the analysis is made in Table 24. The points achieved by each similarity measure are accumulated on each corresponding metric. The whole number of points is 12 points. That is because of having two datasets and two metrics on three values of clustering variable ( $\mathrm{K}=5, \mathrm{~K}=10$, and $\mathrm{K}$ equal the number of actual classes). The bolded values in Table 24 suggest the highest rank.

Table 24. Clustering Process - Points and Rank of Similarity Measures

Peer] Comput. Sci. reviewing PDF | (CS-2020:12:56084:2:0:NEW 10 Jun 2021) 
737

738

739

740

741

742

743

744

745

746

747

748

749

750

751

752

753

754

755

756

757

758

759

760

761

762

763

764

765

766

767

768

769

770

771

772

773

774

775

776

777

\section{Execution Time Analysis}

The time of each measure was accumulated and averaged as given in Figures 13-14. It is clear that all measures share one fact, in particular Ex-Jaccard and SMTP, the run time is increasing sharply as NF increases steadily. Figures 13-14 give the run time of classification on Reuters and web-kb respectively.

Figure 13 Execution Time - Reuters

As it is shown in Figure 13, BLAB-SM followed by Euclidean and Jaccard were the fastest similarity measures with exception when $\mathrm{NF}=3000$ in which KLD was faster. Manhattan followed by cosine and KLD came the next batch of the fastest measures. On the other hand, the slowest measures were SMTP and Ex-Jaccard with Ex-Jaccard being the slowest. Considering Figure 13, BLAB-SM has been the sole measure that meets the ultimate quest of this work in terms of both effectiveness and efficiency at the same time. It is also worth indicating that the BLAB-SM has been as effective as SMTP and much better than Cosine. However, it is maximally efficient comparing with SMTP and more efficient than Cosine. Considering being faster, BLAB-SM could replace SMTP for text classification and clustering effectively and efficiently.

Figure 14 Execution Time - Web-KB

Also, Figure 14 shows that Jaccard, Euclidean, BLAB-SM, and Manhattan were the fastest similarity measures. Interestingly, like Reuters, BLAB-SM confirms it is being fast measure on Web-KB as well. In general, Cosine was faster than KLD yet slower than BLAB-SM. On the other extreme, Ex-Jaccard and SMTP were seen to be slower measures with Ex-Jaccard being the slowest. In conclusion, according to all results drawn in Tables (8-16), and considering Figure 14, BLAB-SM has been the sole measure that meets the ultimate quest of this work in terms of effectiveness and efficiency comparing with SMTP in particular and all measures in general. BLAB-SM has been as effective as SMTP, but maximally efficient compared to SMTP, and it also could replace SMTP and Cosine for text classification effectively and efficiently.

\section{Merits of BLAB-SM}

In this subsection, it is worth referring briefly to the merits of our proposed measures over its competitors chiefly the SMTP. Concisely, the merits could be drawn as follows; (1) BLAB-SM has a simplistic design compared to SMTP. Moreover, BLAB-SM is bounded by upper and lower values in the same design with no complexity being added in the design, SMTP however needed an additional condition to restrict values of SMTP between zero and one (Kumar Nagwani 2014). (2) BLAB-SM has been shown as effective as SMTP (and even better as it is the case on $\mathrm{Web}-\mathrm{KB}$ ) on the high dimensional dataset as all results asserted this claim when NF grew and exceeded 6000 features on both datasets compared to SMTP. In other words, they both

Peer] Comput. Sci. reviewing PDF | (CS-2020:12:56084:2:0:NEW 10 Jun 2021) 
778 have an almost equal performance trend. Finally, (3) BLAB-SM has been observed to be 779 impressively efficient compared to SMTP in particular and all state-of-art measures in general.

780 Time Tables and graphs asserted this claim (see Table 25). When running measures on all 781 features, the BLAB-SM has barely reached 29 minutes on Reuters and 23 minutes on web-KB 782 compared to SMTP which needed roughly 804.933 minutes on Reuters and 336.567 minutes on 783 Web-KB respectively. It is also noted that BLAB-SM is significantly faster than cosine which 784 needed roughly 58.28 minutes on Reuters and 26.37 minutes on Web-KB respectively. With the 785 BLAB-SM is being high-speed measure of a competitive performance, BLAB-SM could be

786

787

788

789

790

791

792

793

794

795

796

797

798

799

800

801

802

803

804

805

806

807

808

809

810

811

812

813

814

815

816

817 818 nominated to replace Cosine and SMTP for the text-based applications, chiefly for the big "large-scale" datasets. The run time has been recorded when all features of both datasets were being considered. Table 25 summarizes the run time of all measures along with the BLAB-SM's improvement Rate against these measures.

Table 25. Comparison of Classification Run Time (in Minutes) of BLAB-SM against all Measures on both Datasets

\section{CONCLUSIONS AND FUTURE WORK}

This work has developed a new similarity measure called Boolean Logic Algebra-Based Similarity measure (BLAB-SM) for the text-based applications. BLAB-SM has been designed to logically treat and process documents the same way the Boolean gates work. In the process of developing our BLAB-SM measure, a thorough experimental study has been conducted for several similarity measures for text classification and clustering. The results conclusively showed that the BLAB-SM similarity measure achieved a highly competitive performance on text retrieval quality (classification and clustering) and run-time efficiency. The experimental results, revealed that BLAB-SM, SMTP, and Cosine scored the highest performance trends (with BLAB-SM and SMTP being the top performers) compared to Ex-Jaccard, Euclidean, Manhattan, and kullback Leibler measures. Ex-Jaccard showed a competitive performance on Reuters, though. On the other hand, Manhattan and kullback Leibler were seen to have the worst results. However, Ex-Jaccard and Euclidean had shown a fluctuating performance. They could be considered as middle-ground solutions between the best and worst measures with Ex-Jaccard being superior to Euclidean.

Jaccard has been experimentally proven ineffective choice when dealing with TF-IDF-based document matching. Given this fact, Jaccard was excluded from any further comparison with the state-of-the-art measures. That is because Jaccard depends basically on the rate of common features which is far less in the TF-IDF VSM matrix. Nevertheless, Jaccard has long been an effective measure when dealing with BoW based document matching. However, Jaccard showed a good clustering performance. BLAB-SM, Euclidean, SMTP, and Cosine were noted to be the top performers in terms of clustering, with BLAB-SM and Euclidean being the best. 
819 In conclusion, run time comparison showed that BLAB-SM is one of the fastest similarity 820 measures compared to all benchmarked measures in this work, making it highly promising in 821 the machine learning and text mining fields. SMTP and Ex-Jaccard measures had been able to 822 achieve good results; yet, at the cost of their efficiency as they were slow, Ex-Jaccard being the 823 slowest measure. BLAB-SM is significantly efficient compared to SMTP while both have 824 constantly been the most effective similarity measures.

825

The follow-up work is planned to consider the semantic aspect along with including other similarity measures [Amer \& Abdalla, 2020; Oghbaie \& Mohammadi Zanjireh, 2018; Sohangir \& Wang, 2017; Aryal, Ting, Washio, \& Haffari, 2019) in the experimental study. Extensive experiments are planned to be conducted on a large-scale datasets to verify the conclusions of this work.

\section{ACKNOWLEDGEMENT}

833 The authors would like to thank and appreciate the support received from the Research Office of 834 Zayed University for providing the necessary facilities to accomplish this work. This research 835 has been supported by Research Incentive Fund (RIF) Grant Activity Code: R19093 - Zayed 836 University, UAE.

837

\section{REFERENCES}

Zhang, X., \& Zuo, J. (2019). Measuring similarity between sequential datasets. In ACM International Conference Proceeding Series. Association for Computing Machinery. https://doi.org/10.1145/3321408.3322844

Gweon, H., Schonlau, M., \& Steiner, S. H. A. (2019). The k conditional nearest neighbor algorithm for classification and class probability estimation. PeerJ Computer Science, 2019(5). https://doi.org/10.7717/peerj-cs.194

Amer, A. A. (2020). On K-means clustering-based approach for DDBSs design. Journal of Big Data, 7(1). https://doi.org/10.1186/s40537-02000306-9

Kogan, J., Teboulle, M., \& Nicholas, C. (2005). Data driven similarity measures for k-means like clustering algorithms. Information Retrieval, 8(2), 331-349. https://doi.org/10.1007/s10791-005-5666-8

Amer, A. A., Abdalla, H. I., \& Nguyen, L. (2021). Enhancing recommendation systems performance using highly-effective similarity measures. Knowledge-Based Systems, 106842. https://doi.org/10.1016/j.knosys.2021.106842.

Amer, A. A., \& Abdalla, H. I. (2020). A set theory based similarity measure for text clustering and classification. Journal of Big Data, 7(1). https://doi.org/10.1186/s40537-020-00344-3

Rachkovskij, D. A. (2017). Binary Vectors for Fast Distance and Similarity Estimation. Cybernetics and Systems Analysis, 53(1), $138-156$. https://doi.org/10.1007/s10559-017-9914-x

Gweon, H., Schonlau, M., \& Steiner, S. H. B. (2019). Nearest labelset using double distances for multi-label classification. PeerJ Computer Science, 5. https://doi.org/10.7717/PEERJ-CS.242

Kanungo, T., Mount, D. M., Netanyahu, N. S., Piatko, C. D., Silverman, R., \& Wu, A. Y. (2002). An efficient k-means clustering algorithms: Analysis and implementation. IEEE Transactions on Pattern Analysis and Machine Intelligence, $24(7), 881-892$. https://doi.org/10.1109/TPAMI.2002.1017616.

Holzinger, A., Schantl, J., Schroettner, M., Seifert, C., \& Verspoor, K. (2014). Biomedical text mining: State-of-the-art, open problems and future challenges. Lecture Notes in Computer Science (Including Subseries Lecture Notes in Artificial Intelligence and Lecture Notes in Bioinformatics), 8401, 271-300. https://doi.org/10.1007/978-3-662-43968-5_16.

Afzali, M., \& Kumar, S. (2017). Comparative Analysis of Various Similarity Measures for Finding Similarity of Two Documents. International Journal of Database Theory and Application, 10(2), 23-30. https://doi.org/10.14257/ijdta.2017.10.2.02.

Oghbaie, M., \& Mohammadi Zanjireh, M. (2018). Pairwise document similarity measure based on present term set. Journal of Big Data, 5(1). https://doi.org/10.1186/s40537-018-0163-2

Sohangir, S., \& Wang, D. (2017). Improved sqrt-cosine similarity measurement. Journal of Big Data, 4(1). https://doi.org/10.1186/s40537-0170083-6

Lin, Y. S., Jiang, J. Y., \& Lee, S. J. (2014). A similarity measure for text classification and clustering. IEEE Transactions on Knowledge and Data Engineering, 26(7), 1575-1590. https://doi.org/10.1109/TKDE.2013.19

Shahmirzadi, O., Lugowski, A., \& Younge, K. (2019). Text similarity in vector space models: A comparative study. In Proceedings - 18th IEEE International Conference on Machine Learning and Applications, ICMLA 2019 (pp. 659-666). Institute of Electrical and Electronics Engineers Inc. https://doi.org/10.1109/ICMLA.2019.00120

Ke, W. (2017). Text retrieval based on least information measurement. In ICTIR 2017 - Proceedings of the 2017 ACM SIGIR International 
873

874

875

Conference on the Theory of Information Retrieval (pp. 125-132). Association for Computing Machinery, Inc. https://doi.org/10.1145/3121050.3121075

White, R. W., \& Jose, J. M. (2004). A study of topic similarity measures. In Proceedings of Sheffield SIGIR - Twenty-Seventh Annual International ACM SIGIR Conference on Research and Development in Information Retrieval (pp. 520-521). Association for Computing Machinery (ACM). https://doi.org/10.1145/1008992.1009100

Lakshmi, R., \& Baskar, S. (2021). Efficient text document clustering with new similarity measures. International Journal of Business Intelligence and Data Mining, 18(1), 109-126. https://doi.org/10.1504/IJBIDM.2021.111741.

Kotte, V. K., Rajavelu, S., \& Rajsingh, E. B. (2020). A Similarity Function for Feature Pattern Clustering and High Dimensional Text Document Classification. Foundations of Science, 25(4), 1077-1094. https://doi.org/10.1007/s10699-019-09592

Thompson, V. U., Panchev, C., \& Oakes, M. (2015). Performance evaluation of similarity measures on similar and dissimilar text retrieval. In IC3K 2015 - Proceedings of the 7th International Joint Conference on Knowledge Discovery, Knowledge Engineering and Knowledge Management (Vol. 1, pp. 577-584). SciTePress. https://doi.org/10.5220/0005619105770584

Robertson, S. (2004). Understanding inverse document frequency: On theoretical arguments for IDF. Journal of Documentation, 60(5), 503-520. https://doi.org/10.1108/00220410410560582

Zhao, R., \& Mao, K. (2018). Fuzzy Bag-of-Words Model for Document Representation. IEEE Transactions on Fuzzy Systems, 26(2), $794-804$. https://doi.org/10.1109/TFUZZ.2017.2690222

Joulin, A., Grave, E., Bojanowski, P., \& Mikolov, T. (2017). Bag of tricks for efficient text classification. In 15th Conference of the European Chapter of the Association for Computational Linguistics, EACL 2017 - Proceedings of Conference (Vol. 2, pp. 427-431). Association for Computational Linguistics (ACL). https://doi.org/10.18653/v1/e17-2068

Heidarian, A., \& Dinneen, M. J. (2016). A Hybrid Geometric Approach for Measuring Similarity Level among Documents and Document Clustering. In Proceedings - 2016 IEEE 2nd International Conference on Big Data Computing Service and Applications, BigDataService 2016 (pp. 142-151). Institute of Electrical and Electronics Engineers Inc. https://doi.org/10.1109/BigDataService.2016.14

Cordeiro De, Amorim, R., \& Mirkin, B. (2012). Minkowski metric, feature weighting and anomalous cluster initializing in K-Means clustering. Pattern Recognition, 45(3), 1061-1075. https://doi.org/10.1016/j.patcog.2011.08.012

Kumar, V., Chhabra, J. K., \& Kumar, D. (2014). Performance Evaluation of Distance Metrics in the Clustering Algorithms. INFOCOMP, 13(1), $38-51$.

François, D., Wertz, V., \& Verieysen, M. (2007). The concentration of fractional distances. IEEE Transactions on Knowledge and Data Engineering, 19(7), 873-886. https://doi.org/10.1109/TKDE.2007.1037

D’hondt, J., Vertommen, J., Verhaegen, P. A., Cattrysse, D., \& Duflou, J. R. (2010). Pairwise-adaptive dissimilarity measure for document clustering. Information Sciences, 180(12), 2341-2358. https://doi.org/10.1016/j.ins.2010.02.021

Li, X., Yao, C., Fan, F., \& Yu, X. (2017). A text similarity measurement method based on singular value decomposition and semantic relevance. Journal of Information Processing Systems, 13(4), 863-875. https://doi.org/10.3745/JIPS.02.0067

Kullback, S., \& Leibler, R. A. (1951). On Information and Sufficiency. The Annals of Mathematical Statistics, 22(1), 79-86. https://doi.org/10.1214/aoms/1177729694

Subhashini, R., \& Kumar, V. J. S. (2010). Evaluating the performance of similarity measures used in document clustering and information retrieval. In Proceedings - 1st International Conference on Integrated Intelligent Computing, ICIIC 2010 (pp. 27-31). https://doi.org/10.1109/ICIIC.2010.42

Li, B., \& Han, L. (2013). Distance weighted cosine similarity measure for text classification. In Lecture Notes in Computer Science (including subseries Lecture Notes in Artificial Intelligence and Lecture Notes in Bioinformatics) (Vol. 8206 LNCS, pp. 611-618). https://doi.org/10.1007/978-3-642-41278-3 74.

Arivarasan, A., \& Karthikeyan, M. (2019). Data mining K-means document clustering using tfidf and word frequency count. International Journal of Recent Technology and Engineering, 8(2), 2542-2549. https://doi.org/10.35940/ijrte.B1718.078219

Zhao, Y., \& Karypis, G. (2002). Comparison of Agglomerative and Partitional Document Clustering Algorithms. Performance Computing, 1-11.

Aslam, J. A., \& Frost, M. (2003). An Information-theoretic Measure for Document Similarity. In SIGIR Forum (ACM Special Interest Group on Information Retrieval) (pp. 449-450). Association for Computing Machinery. https://doi.org/10.1145/860500.860545

Tata, S., \& Patel, J. M. (2007). Estimating the selectivity of tf-idf based cosine similarity predicates. In SIGMOD Record (Vol. 36, pp. 75-80). https://doi.org/10.1145/1361348.1361351

Tanimoto T.. (1957). An elementary mathematical theory of classification and prediction. IBM, Armonk, NY, USA, Tech. Rep. IBM Int. Rep.

Haroutunian, E. (2011). Information Theory and Statistics. In International Encyclopedia of Statistical Science (pp. 666-667). Springer Berlin Heidelberg. https://doi.org/10.1007/978-3-642-04898-2_643

Amigó, E., Giner, F., Gonzalo, J., \& Verdejo, F. (2020). On the foundations of similarity in information access. Information Retrieval Journal, 23(3), 216-254. https://doi.org/10.1007/s10791-020-09375

Kumar Nagwani, N. (2015, September 1). A Comment on "A Similarity Measure for Text Classification and Clustering." IEEE Transactions on Knowledge and Data Engineering. IEEE Computer Society. https://doi.org/10.1109/TKDE.2015.2451616

Aryal, S., Ting, K. M., Washio, T., \& Haffari, G. (2019), February 9). A new simple and effective measure for bag-of-word inter-document similarity measurement. ArXiv. ArXiv 
Figure 1

Figure 1 AND Gate

Figure 1 AND Gate 


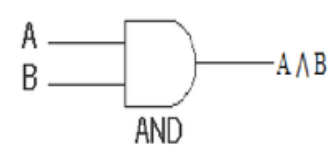

Figure 1 AND Gate 
Figure 2

Figure 2 OR Gate

Figure 2 OR Gate 


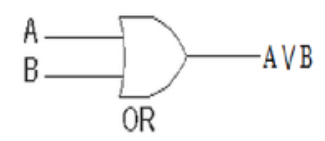

Figure 2 OR Gate 
Figure 3

Figure 3 EXOR Gate

Figure 3 EXOR Gate 


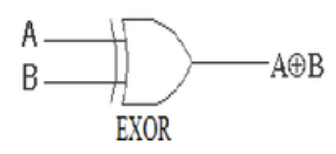

Figure 3 EXOR Gate 
Figure 4

Figure 4. The experimental Design Flow Chart

Figure 4. The experimental Design Flow Chart 

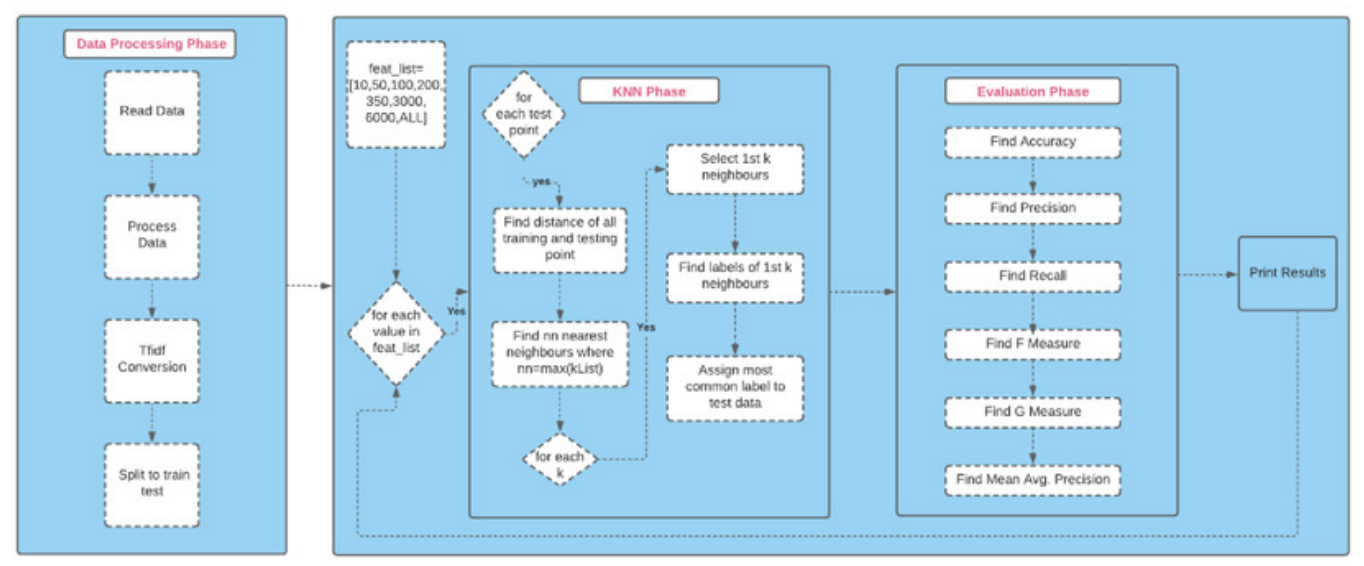


\section{Figure 5}

Figure 5 Accuracy over all Measures on all NF values - Reuters

Figure 5 Accuracy over all Measures on all NF values- Average results $(K=1-120 ;+2)-$ Reuters 


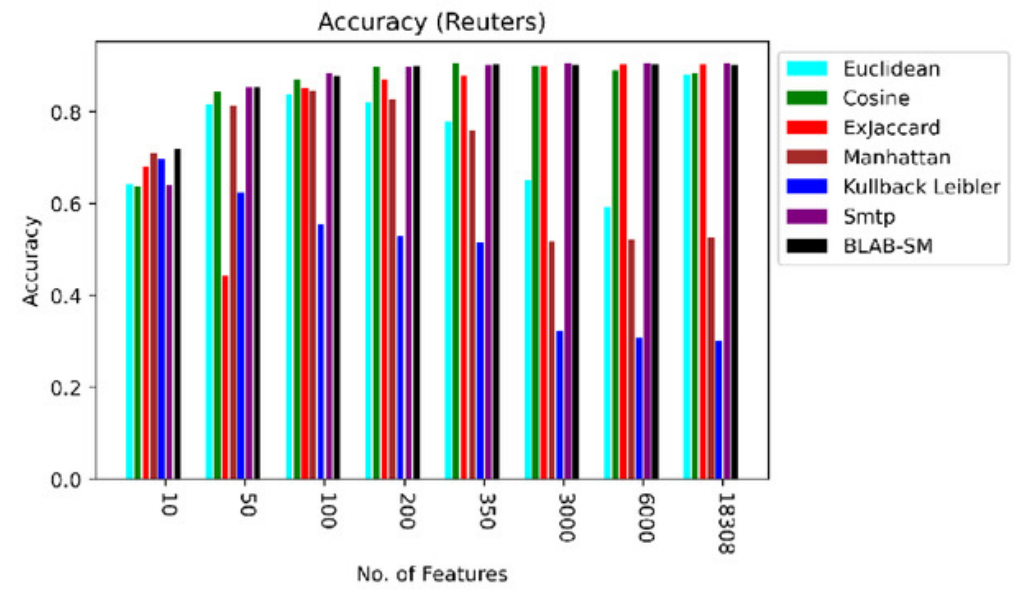

Figure 4 Accuracy over all Measures on all NF values- Average results $(K=1-120 ;+2)-$ Reuters 


\section{Figure 6}

Figure 6 F-measure over all Measures on all NF values- Reuters

Figure 6 F-measure over all Measures on all NF values- Average results $(K=1-120 ;+2)$ Reuters 


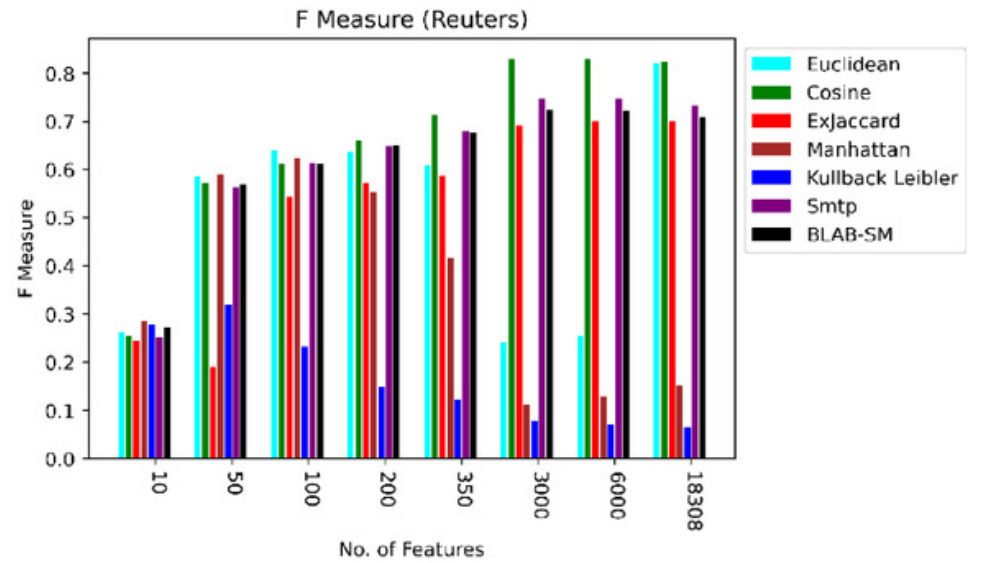

Figure 5 F-measure over all Measures on all NF values- Average results $(\mathrm{K}=1-120 ;+2)$ - Reuters 


\section{Figure 7}

Figure 7 AMP over all Measures on all NF values- Reuters

Figure 7 AMP over all Measures on all NF values- Average results $(K=1-120 ;+2)$ - Reuters 


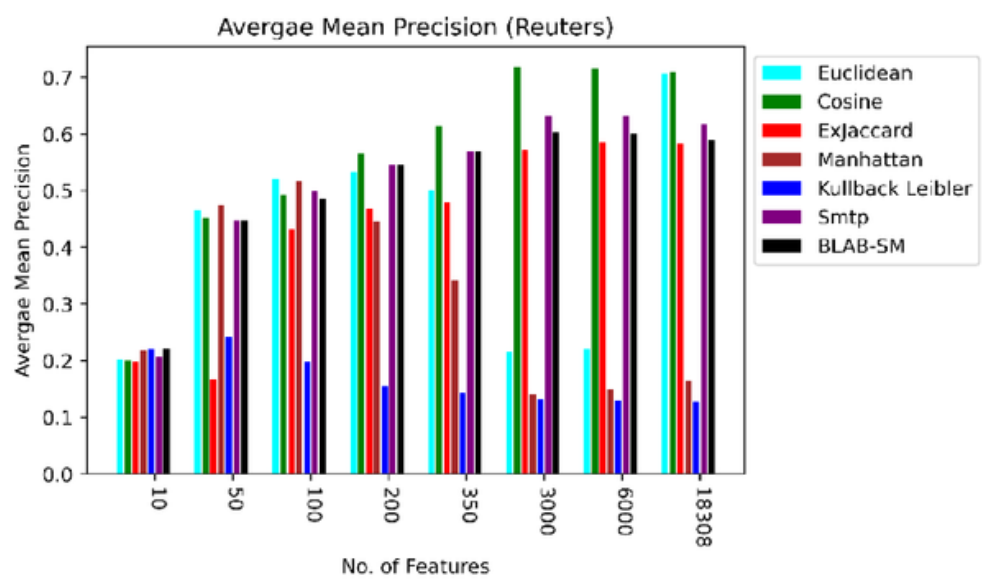

Figure 6 AMP over all Measures on all NF values- Average results $(\mathrm{K}=1-120 ;+2)$ - Reuters 
Figure 8

Figure 8. Accuracy over all Measures on all NF values - Web-KB

Figure 8. Accuracy over all Measures on all NF values- Average results $(K=1-120 ;+2)-$ WebKB 


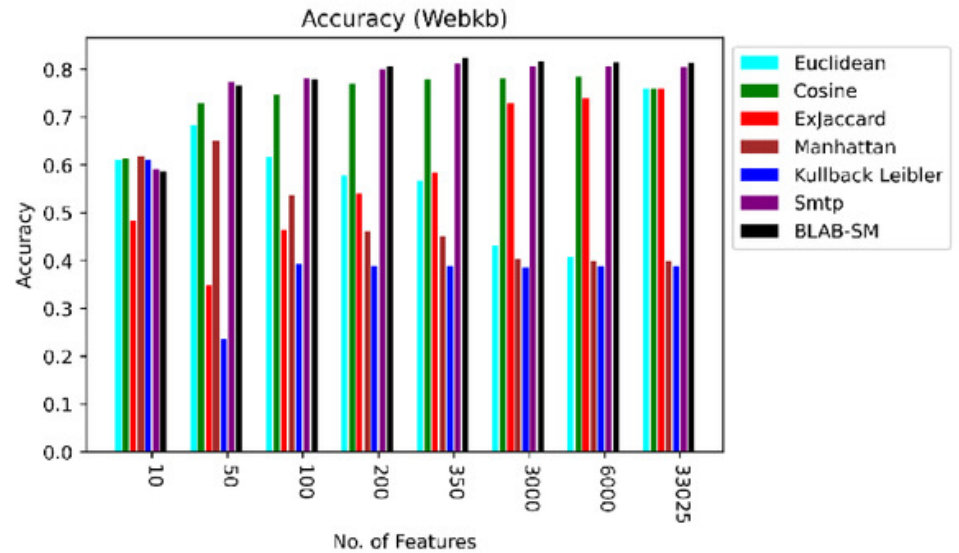

Figure 7. Accuracy over all Measures on all NF values- Average results $(K=1-120 ;+2)-$ Web-KB 


\section{Figure 9}

Figure 9 F-measure over all Measures on all NF values-webkb

Figure 9 F-measure over all Measures on all NF values- Average results $(K=1-120 ;+2)$ Web-KB 


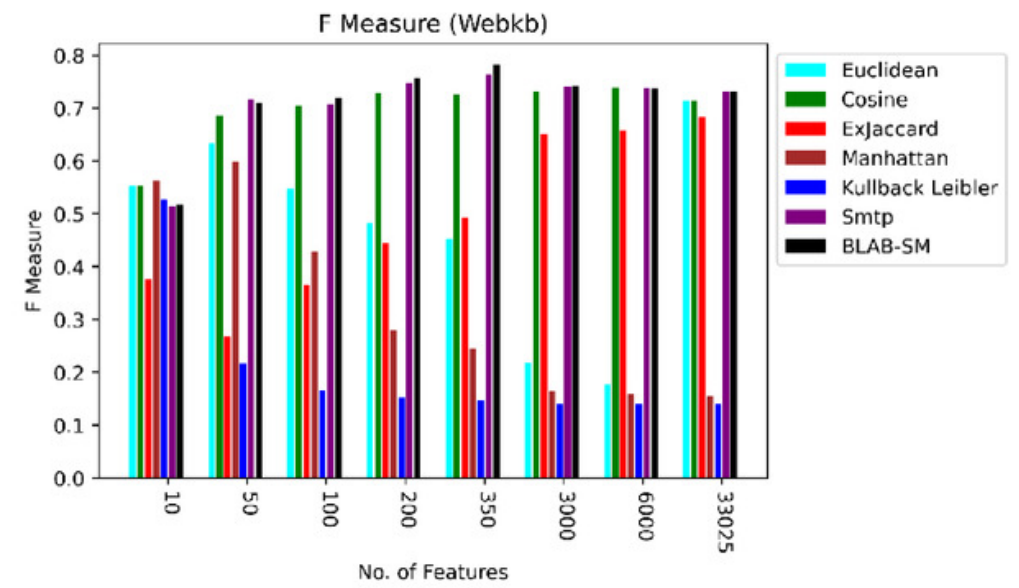

Figure 8 F-measure over all Measures on all NF values- Average results $(K=1-120 ;+2)-$ Web-KB 


\section{Figure 10}

Figure 10 AMP over all Measures on all NF values-webkb

Figure 10 AMP over all Measures on all NF values- Average results $(K=1-120 ;+2)-$ Web-KB 


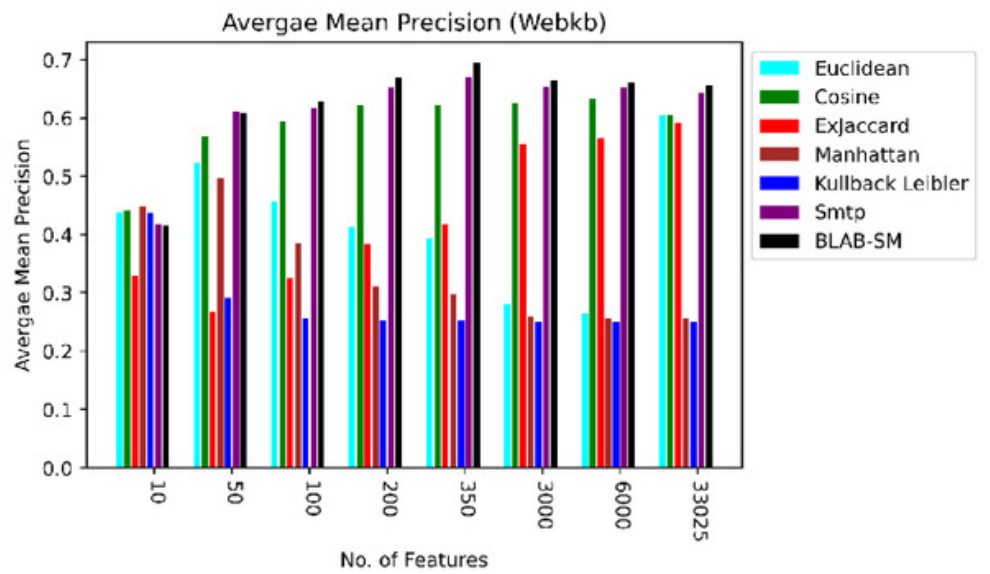

Figure 9 AMP over all Measures on all NF values- Average results $(\mathrm{K}=1-120 ;+2)-\mathrm{Web}-\mathrm{KB}$ 


\section{Figure 11}

Figure 11. Performance of all Measures on both datasets - Reuters

Figure 11. Performance of all Measures on both datasets - Reuters 


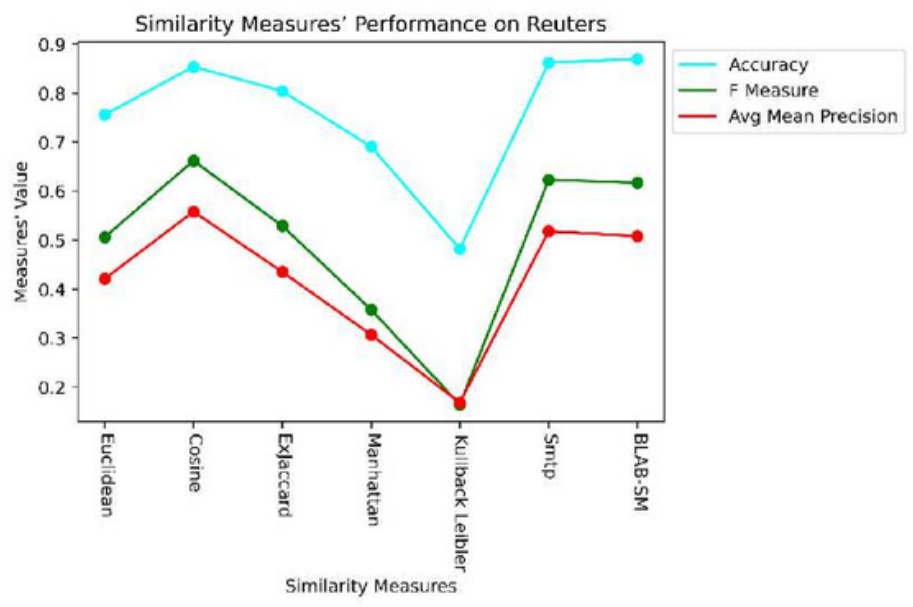

Figure 10. Performance of all Measures on both datasets - Reuters 


\section{Figure 12}

Figure 12. Performance of all Measures on both datasets -Web-KB

Figure 12. Performance of all Measures on both datasets -Web-KB 


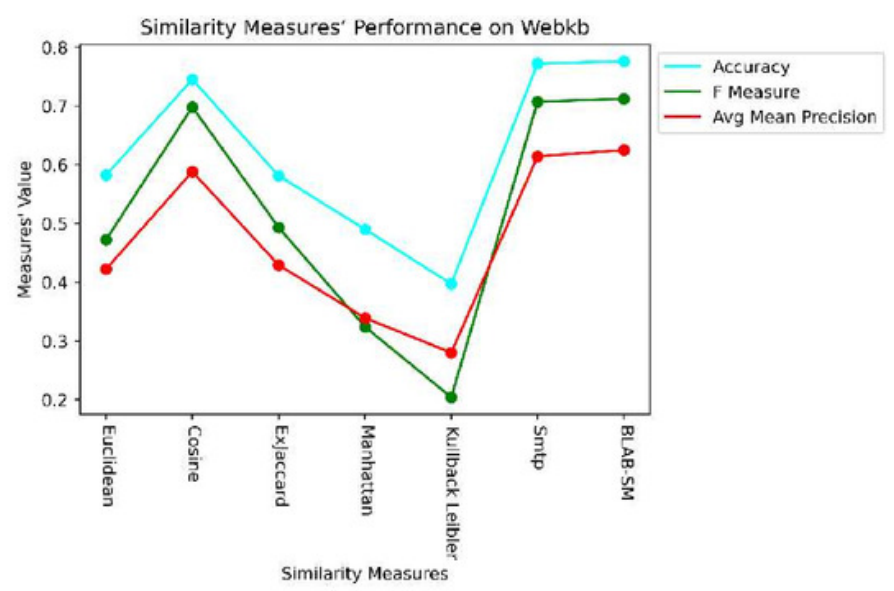

Figure 11. Performance of all Measures on both datasets -Web-KB 
Figure 13

Figure 13 Execution Time - Reuters

Figure 13 Execution Time - Reuters 


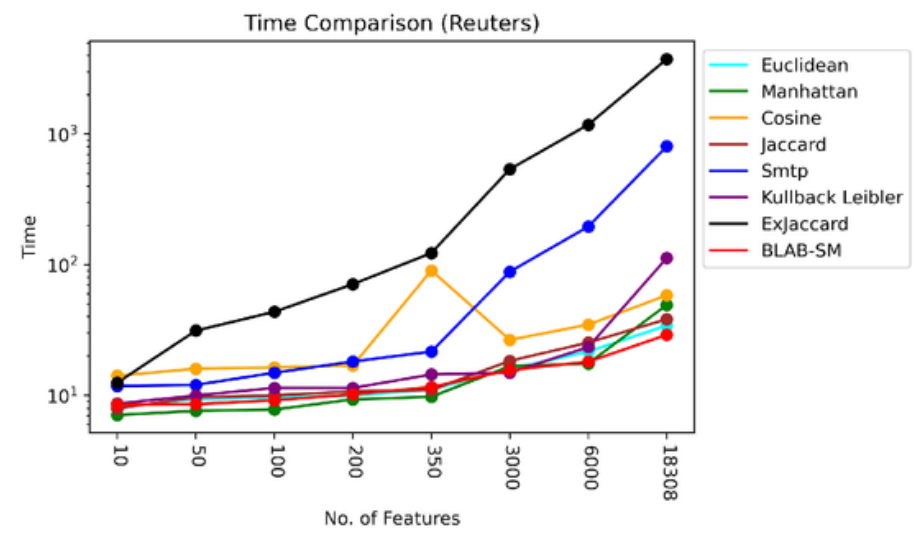

Figure 12 Execution Time - Reuters 
Figure 14

Figure 14 Execution Time - Web-KB

Figure 14 Execution Time - Web-KB 


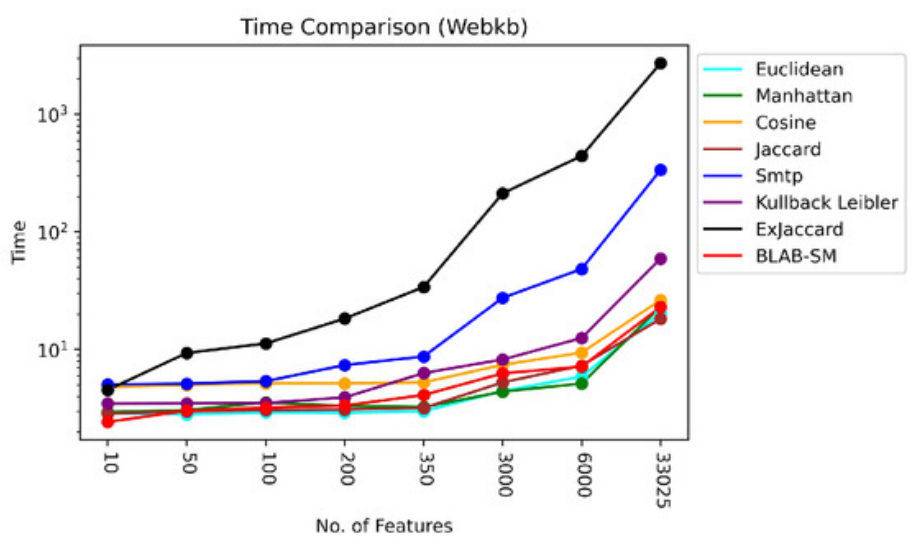

Figure 13 Execution Time - Web-KB 


\section{Table $\mathbf{1}$ (on next page)}

Table 1 A AND B table truth

Table 1 A AND B table truth 
1

Table 1 A AND B table truth
A B $A \wedge B$
$0 \quad 0 \quad 0$
$\begin{array}{lll}0 & 1 & 0\end{array}$ 


\section{Table 2 (on next page)}

Table 2 A OR B table truth

Table 2 A OR B table truth 
1

2

3

4

Table 2 A OR B table truth
A B $\quad$ $\vee$ B
$\begin{array}{lll}0 & 0 & 0\end{array}$
$0 \quad 1 \quad 1$ 


\section{Table $\mathbf{3}$ (on next page)}

Table 3 A EXOR B table truth

Table 3 A EXOR B table truth 
1

2

3

4

Table 3 A EXOR B table truth
A $\quad \mathrm{B} \quad \mathrm{A} \oplus \mathrm{B}$
$\begin{array}{lll}0 & 0 & 0\end{array}$
$\begin{array}{lll}0 & 1 & 1\end{array}$

PeerJ Comput. Sci. reviewing PDF | (CS-2020:12:56084:2:0:NEW 10 Jun 2021) 


\section{Table 4 (on next page)}

Table 4 Splitting of documents among four classes in Web-KB dataset

Table 4 Splitting of documents among four classes in Web-KB dataset 
1

2

3

4

5

6

Table 4 Splitting of documents among four classes in Web-KB dataset

\begin{tabular}{cc}
\hline Class & Samples \\
\hline Project & 504 \\
Course & 930 \\
Student & 1641 \\
Faculty & 1124 \\
Total & 4199 \\
\hline
\end{tabular}




\section{Table 5 (on next page)}

Table 5 Splitting of documents among eight classes in Reuters-R8 dataset

Table 5 Splitting of documents among eight classes in Reuters-R8 dataset 
1

2

3

4

5

Table 5 Splitting of documents among eight classes in Reuters-R8 dataset

\begin{tabular}{cc}
\hline Class & Samples \\
\hline Cq & 2292 \\
Crude & 374 \\
Earn & 3923 \\
Grain & 51 \\
Interest & 271 \\
Money-fix & 293 \\
Ship & 144 \\
Trade & 326 \\
Total & 7674 \\
\hline
\end{tabular}




\section{Table 6(on next page)}

Table 6. Performance Evaluation of all Measures when, $\mathrm{NF}=10$ - the averaged results $(\mathrm{K}=1-120 ;+2)$

Table 6. Performance Evaluation of all Measures when, $\mathrm{NF}=10$ - the averaged results $(\mathrm{K}=1-120 ;+2)$ 
1

2

3

4

Table 6. Performance Evaluation of all Measures when, $\mathrm{NF}=10$ - the averaged results $(\mathrm{K}=1-120 ;+2)$

\begin{tabular}{|c|c|c|c|c|c|c|c|c|c|c|c|c|}
\hline \multirow{2}{*}{$\begin{array}{c}\text { Dataset } \\
\text { Similarity/ } \\
\text { Criterion }\end{array}$} & \multicolumn{6}{|c|}{ Reuters-8 } & \multicolumn{6}{|c|}{ Web-KB } \\
\hline & $\mathrm{ACC}$ & PRE & REC & FM & GM & AMP & ACC & PRE & REC & FM & GM & AMP \\
\hline Euclidean & 0.642 & 0.345 & 0.272 & 0.262 & 0.504 & 0.203 & 0.611 & 0.598 & 0.540 & 0.553 & 0.679 & 0.438 \\
\hline Cosine & 0.638 & 0.282 & 0.266 & 0.255 & 0.499 & 0.199 & 0.614 & 0.592 & 0.542 & 0.553 & 0.682 & 0.442 \\
\hline Ex-Jaccard & 0.680 & 0.278 & 0.254 & 0.244 & 0.487 & 0.197 & 0.484 & 0.454 & 0.401 & 0.377 & 0.569 & 0.329 \\
\hline Manhattan & 0.710 & 0.344 & 0.288 & 0.286 & 0.521 & 0.218 & 0.618 & 0.620 & 0.548 & 0.562 & 0.685 & 0.448 \\
\hline KLD & 0.696 & 0.361 & 0.294 & 0.277 & 0.525 & 0.219 & 0.612 & 0.629 & 0.523 & 0.527 & 0.669 & 0.437 \\
\hline SMTP & 0.641 & 0.300 & 0.271 & 0.252 & 0.503 & 0.206 & 0.590 & 0.576 & 0.516 & 0.514 & 0.661 & 0.417 \\
\hline BLAB-SM & 0.720 & 0.303 & 0.285 & 0.272 & 0.519 & 0.221 & 0.587 & 0.584 & 0.516 & 0.517 & 0.661 & 0.415 \\
\hline
\end{tabular}




\section{Table 7 (on next page)}

Table 7. Performance Evaluation of all Measures when, $\mathrm{NF}=50$ - the averaged results $(\mathrm{K}=1-120 ;+2)$

Table 7. Performance Evaluation of all Measures when, $\mathrm{NF}=50$ - the averaged results $(\mathrm{K}=1-120 ;+2)$ 
1

2

3

4

5

Table 7. Performance Evaluation of all Measures when, $\mathrm{NF}=50$ - the averaged results $(\mathrm{K}=1-120 ;+2)$

\begin{tabular}{cccccccccccccc}
\hline Dataset & \multicolumn{1}{c}{ Reuters-8 } & \multicolumn{1}{c}{ Web-KB } \\
\hline Similarity/ & ACC & PRE & REC & FM & GM & AMP & ACC & PRE & REC & FM & GM & AMP \\
Criterion & & & & & & & & & & \\
Euclidean & 0.817 & 0.635 & 0.572 & 0.586 & 0.745 & 0.465 & 0.684 & 0.747 & 0.605 & 0.634 & 0.728 & 0.522 \\
Cosine & 0.843 & 0.654 & 0.552 & 0.572 & 0.732 & 0.452 & 0.729 & 0.742 & 0.669 & 0.687 & 0.776 & 0.568 \\
Ex-Jaccard & 0.442 & 0.310 & 0.181 & 0.190 & 0.399 & 0.167 & 0.348 & 0.349 & 0.281 & 0.269 & 0.461 & 0.268 \\
Manhattan & 0.813 & 0.642 & 0.586 & 0.591 & 0.753 & 0.475 & 0.652 & 0.777 & 0.565 & 0.598 & 0.697 & 0.498 \\
KLD & 0.6245 & 0.602 & 0.282 & 0.320 & 0.505 & 0.242 & 0.236 & 0.527 & 0.316 & 0.217 & 0.496 & 0.292 \\
SMTP & 0.853 & 0.647 & 0.558 & 0.563 & 0.737 & 0.447 & 0.774 & 0.803 & 0.694 & 0.716 & 0.796 & 0.611 \\
BLAB-SM & 0.853 & 0.650 & 0.548 & 0.569 & 0.730 & 0.447 & 0.766 & 0.820 & 0.682 & 0.709 & 0.787 & 0.609 \\
\hline
\end{tabular}




\section{Table 8(on next page)}

Table 8. Performance Evaluation of all Measures when, $\mathrm{NF}=100$ - the averaged results $(\mathrm{K}=1-120 ;+2)$

Table 8. Performance Evaluation of all Measures when, $\mathrm{NF}=100$ - the averaged results $(\mathrm{K}=1-120 ;+2)$ 
1

2

3

4

5

6

Table 8. Performance Evaluation of all Measures when, $\mathrm{NF}=100-$ the averaged results $(\mathrm{K}=1-120 ;+2)$

\begin{tabular}{cccccccccccccc}
\hline Dataset & \multicolumn{1}{c}{ Reuters } & \multicolumn{1}{c}{ Web-KB } \\
\hline Similarity/ & ACC & PRE & REC & FM & GM & AMP & ACC & PRE & REC & FM & GM & AMP \\
Criterion & & & & & & & & & & \\
Euclidean & 0.839 & 0.670 & 0.644 & 0.640 & 0.792 & 0.521 & 0.617 & 0.729 & 0.525 & 0.548 & 0.666 & 0.456 \\
Cosine & 0.870 & 0.686 & 0.592 & 0.610 & 0.760 & 0.493 & 0.747 & 0.776 & 0.685 & 0.705 & 0.788 & 0.593 & \\
Ex-Jaccard & 0.852 & 0.632 & 0.523 & 0.544 & 0.713 & 0.433 & 0.464 & 0.555 & 0.372 & 0.365 & 0.544 & 0.325 \\
Manhattan & 0.847 & 0.686 & 0.638 & 0.624 & 0.788 & 0.517 & 0.536 & 0.772 & 0.426 & 0.429 & 0.587 & 0.386 \\
KLD & 0.555 & 0.659 & 0.211 & 0.232 & 0.432 & 0.197 & 0.394 & 0.386 & 0.260 & 0.166 & 0.442 & 0.256 \\
SMTP & 0.884 & 0.667 & 0.603 & 0.614 & 0.768 & 0.499 & 0.781 & 0.815 & 0.692 & 0.708 & 0.796 & 0.617 \\
BLAB-SM & 0.879 & 0.669 & 0.585 & 0.610 & 0.756 & 0.486 & 0.778 & 0.853 & 0.693 & 0.720 & 0.795 & 0.629 \\
\hline
\end{tabular}




\section{Table 9 (on next page)}

Table 9. Performance Evaluation of all Measures when, $\mathrm{NF}=200$ - the averaged results $(\mathrm{K}=1-120 ;+2)$

Table 9. Performance Evaluation of all Measures when, $\mathrm{NF}=200$ - the averaged results $(\mathrm{K}=1-120 ;+2)$ 
Table 9. Performance Evaluation of all Measures when, $\mathrm{NF}=200-$ the averaged results $(\mathrm{K}=1-120 ;+2)$

\begin{tabular}{|c|c|c|c|c|c|c|c|c|c|c|c|c|}
\hline \multirow{2}{*}{$\begin{array}{c}\text { Dataset } \\
\text { Similarity/ } \\
\text { Criterion }\end{array}$} & \multicolumn{6}{|c|}{ Reuters } & \multicolumn{6}{|c|}{ Web-KB } \\
\hline & $\mathrm{ACC}$ & PRE & REC & $\mathrm{FM}$ & GM & AMP & $\mathrm{ACC}$ & PRE & REC & FM & GM & AMP \\
\hline Euclidean & 0.821 & 0.738 & 0.607 & 0.637 & 0.766 & 0.533 & 0.578 & 0.742 & 0.470 & 0.482 & 0.624 & 0.414 \\
\hline Cosine & 0.897 & 0.739 & 0.642 & 0.661 & 0.793 & 0.565 & 0.770 & 0.797 & 0.710 & 0.729 & 0.806 & 0.621 \\
\hline Ex-Jaccard & 0.871 & 0.644 & 0.554 & 0.572 & 0.735 & 0.468 & 0.540 & 0.638 & 0.443 & 0.446 & 0.604 & 0.383 \\
\hline Manhattan & 0.828 & 0.733 & 0.510 & 0.553 & 0.700 & 0.446 & 0.461 & 0.792 & 0.327 & 0.280 & 0.504 & 0.311 \\
\hline KLD & 0.530 & 0.611 & 0.161 & 0.149 & 0.375 & 0.155 & 0.389 & 0.350 & 0.254 & 0.152 & 0.437 & 0.253 \\
\hline SMTP & 0.897 & 0.721 & 0.635 & 0.648 & 0.789 & 0.545 & 0.800 & 0.839 & 0.725 & 0.748 & 0.818 & 0.651 \\
\hline BLAB-SM & 0.900 & 0.719 & 0.629 & 0.650 & 0.785 & 0.545 & 0.807 & 0.874 & 0.727 & 0.757 & 0.820 & 0.669 \\
\hline
\end{tabular}




\section{Table $\mathbf{1 0}$ (on next page)}

Table 10 Performance Evaluation of all Measures when, $\mathrm{NF}=350$ - the averaged results $(\mathrm{K}=1-120 ;+2)$

Table 10 Performance Evaluation of all Measures when, $\mathrm{NF}=350$ - the averaged results $(\mathrm{K}=1-120 ;+2)$ 
1

2

Table 10 Performance Evaluation of all Measures when, $\mathrm{NF}=350$ - the averaged results $(\mathrm{K}=1-120 ;+2)$

\begin{tabular}{cccccccccccccc}
\hline Dataset & \multicolumn{1}{c}{ Reuters } & \multicolumn{1}{c}{ Web-KB } \\
\hline $\begin{array}{c}\text { Similarity/ } \\
\text { Criterion }\end{array}$ & ACC & PRE & REC & FM & GM & AMP & ACC & PRE & REC & FM & GM & AMP \\
Euclidean & 0.780 & 0.785 & 0.551 & 0.608 & 0.727 & 0.501 & 0.567 & 0.737 & 0.451 & 0.452 & 0.610 & 0.394 \\
Cosine & 0.905 & 0.772 & 0.686 & 0.714 & 0.820 & 0.613 & 0.778 & 0.797 & 0.711 & 0.726 & 0.809 & 0.621 \\
Ex-Jaccard & 0.880 & 0.732 & 0.571 & 0.588 & 0.747 & 0.479 & 0.583 & 0.674 & 0.486 & 0.492 & 0.639 & 0.417 \\
Manhattan & 0.760 & 0.755 & 0.368 & 0.417 & 0.583 & 0.341 & 0.451 & 0.618 & 0.310 & 0.245 & 0.490 & 0.297 \\
KLD & 0.516 & 0.548 & 0.146 & 0.123 & 0.357 & 0.142 & 0.388 & 0.368 & 0.252 & 0.147 & 0.435 & 0.252 \\
SMTP & 0.902 & 0.778 & 0.657 & 0.679 & 0.803 & 0.570 & 0.812 & 0.852 & 0.741 & 0.765 & 0.830 & 0.670 \\
BLAB-SM & 0.904 & 0.770 & 0.647 & 0.677 & 0.800 & 0.570 & 0.825 & 0.881 & 0.753 & 0.783 & 0.838 & 0.695 \\
\hline
\end{tabular}




\section{Table $\mathbf{1 1}$ (on next page)}

Table 11 Performance Evaluation of all Measures when, $\mathrm{NF}=3000 \square$ the averaged results $(\mathrm{K}=1-120 ;+2)$

Table 11 Performance Evaluation of all Measures when, $\mathrm{NF}=3000 \square$ the averaged results $(\mathrm{K}=1-120 ;+2)$ 
1

2

3

4

Table 11 Performance Evaluation of all Measures when, NF=3000- the averaged results $(\mathrm{K}=1-120 ;+2)$

\begin{tabular}{|c|c|c|c|c|c|c|c|c|c|c|c|c|}
\hline \multirow{2}{*}{$\begin{array}{c}\text { Dataset } \\
\text { Similarity/ } \\
\text { Criterion }\end{array}$} & \multicolumn{6}{|c|}{ Reuters } & \multicolumn{6}{|c|}{ Web-KB } \\
\hline & $\mathrm{ACC}$ & PRE & REC & FM & GM & AMP & $\mathrm{ACC}$ & PRE & REC & FM & GM & AMP \\
\hline Euclidean & 0.652 & 0.576 & 0.228 & 0.242 & 0.451 & 0.216 & 0.431 & 0.627 & 0.293 & 0.218 & 0.473 & 0.281 \\
\hline Ex-Jaccard & 0.900 & 0.874 & 0.657 & 0.691 & 0.803 & 0.571 & 0.729 & 0.776 & 0.641 & 0.650 & 0.759 & 0.555 \\
\hline Manhattan & 0.517 & 0.224 & 0.141 & 0.112 & 0.350 & 0.140 & 0.404 & 0.286 & 0.263 & 0.165 & 0.446 & 0.260 \\
\hline KLD & 0.323 & 0.245 & 0.135 & 0.077 & 0.345 & 0.132 & 0.386 & 0.174 & 0.250 & 0.141 & 0.433 & 0.250 \\
\hline
\end{tabular}




\section{Table 12 (on next page)}

Table 12. Performance Evaluation of all Measures when, $\mathrm{NF}=6000 \square$ the averaged results $(\mathrm{K}=1-120 ;+2)$

Table 12. Performance Evaluation of all Measures when, $\mathrm{NF}=6000 \square$ the averaged results $(\mathrm{K}=1-120 ;+2)$ 
Table 12. Performance Evaluation of all Measures when, $\mathrm{NF}=6000-$ the averaged results $(\mathrm{K}=1-120 ;+2)$

\begin{tabular}{|c|c|c|c|c|c|c|c|c|c|c|c|c|}
\hline \multirow{2}{*}{$\begin{array}{c}\text { Dataset } \\
\text { Similarity/ } \\
\text { Criterion }\end{array}$} & \multicolumn{6}{|c|}{ Reuters } & \multicolumn{6}{|c|}{ Web-KB } \\
\hline & $\mathrm{ACC}$ & PRE & REC & FM & GM & AMP & $\mathrm{ACC}$ & PRE & REC & FM & GM & AMP \\
\hline Euclidean & 0.593 & 0.714 & 0.227 & 0.253 & 0.446 & 0.220 & 0.407 & 0.448 & 0.269 & 0.177 & 0.451 & 0.265 \\
\hline Cosine & 0.891 & 0.900 & 0.786 & 0.829 & 0.876 & 0.716 & 0.785 & 0.795 & 0.728 & 0.739 & 0.819 & 0.632 \\
\hline Ex-Jaccard & 0.904 & 0.882 & 0.667 & 0.700 & 0.809 & 0.584 & 0.739 & 0.787 & 0.652 & 0.658 & 0.767 & 0.565 \\
\hline Manhattan & 0.521 & 0.327 & 0.151 & 0.129 & 0.362 & 0.150 & 0.398 & 0.263 & 0.260 & 0.159 & 0.443 & 0.257 \\
\hline KLD & 0.308 & 0.254 & 0.131 & 0.069 & 0.339 & 0.130 & 0.387 & 0.176 & 0.251 & 0.140 & 0.434 & 0.250 \\
\hline SMTP & 0.907 & 0.897 & 0.702 & 0.748 & 0.829 & 0.632 & 0.806 & 0.853 & 0.722 & 0.738 & 0.818 & 0.651 \\
\hline BLAB-SM & 0.904 & 0.886 & 0.668 & 0.722 & 0.808 & 0.600 & 0.815 & 0.871 & 0.724 & 0.737 & 0.820 & 0.661 \\
\hline
\end{tabular}




\section{Table $\mathbf{1 3}$ (on next page)}

Table 13. Performance Evaluation of all Measures when, $\mathrm{NF}=$ the whole size (Reuters $=18308$, web- $k b=33025$ features $)-$ the averaged results $(K=1-120 ;+2)$

Table 13. Performance Evaluation of all Measures when, $\mathrm{NF}=$ the whole size (Reuters $=18308$, web- $\mathrm{kb}=33025$ features $)$ - the averaged results $(K=1-120 ;+2)$ 
1

2

3

Table 13. Performance Evaluation of all Measures when, $\mathrm{NF}=$ the whole size (Reuters $=18308$, web-kb=33025 features) - the averaged results $(\mathrm{K}=1-120 ;+2)$

\begin{tabular}{ccccccccccccccc}
\hline Dataset & \multicolumn{1}{c}{ Reuters } \\
\hline Similarity/ & ACC & PRE & REC & FM & GM & AMP & ACC & PRE & REC & FM & GM & AMP \\
Criterion & & & & & & & & & & \\
Euclidean & 0.882 & 0.895 & 0.779 & 0.821 & 0.870 & 0.706 & 0.760 & 0.773 & 0.712 & 0.714 & 0.808 & 0.604 \\
Cosine & 0.884 & 0.898 & 0.779 & 0.823 & 0.871 & 0.709 & 0.760 & 0.773 & 0.712 & 0.714 & 0.808 & 0.604 \\
Ex-Jaccard & 0.904 & 0.883 & 0.666 & 0.700 & 0.809 & 0.583 & 0.760 & 0.800 & 0.679 & 0.685 & 0.787 & 0.591 \\
Manhattan & 0.527 & 0.378 & 0.167 & 0.151 & 0.379 & 0.164 & 0.398 & 0.223 & 0.258 & 0.155 & 0.441 & 0.256 \\
KLD & 0.301 & 0.255 & 0.129 & 0.065 & 0.337 & 0.128 & 0.387 & 0.177 & 0.251 & 0.140 & 0.434 & 0.250 \\
SMTP & 0.905 & 0.894 & 0.688 & 0.733 & 0.821 & 0.617 & 0.804 & 0.851 & 0.718 & 0.730 & 0.816 & 0.644 \\
BLAB-SM & 0.902 & 0.848 & 0.659 & 0.708 & 0.803 & 0.590 & 0.814 & 0.865 & 0.723 & 0.731 & 0.820 & 0.656 \\
\hline
\end{tabular}




\section{Table 14(on next page)}

Table 14. Performance Evaluation of all Measures when the average of averaged results is considered

Table 14. Performance Evaluation of all Measures when the average of averaged results is considered 
1

2

3

Table 14. Performance Evaluation of all Measures when the average of averaged results is considered (Reuters $=18308$, web-kb=33025 features) - the averaged of averaged results $(\mathrm{NF}=10$-All features)

\begin{tabular}{cccccccccccccc}
\hline Dataset & \multicolumn{1}{c}{ Reuters } \\
\hline Similarity/ & ACC & PRE & REC & FM & GM & AMP & ACC & PRE & REC & FM & GM & AMP \\
Criterion & & & & & & & & & & \\
Euclidean & 0.756 & 0.670 & 0.485 & 0.506 & 0.663 & 0.421 & 0.582 & 0.675 & 0.483 & 0.472 & 0.630 & 0.422 \\
Cosine & 0.854 & 0.729 & 0.636 & 0.662 & 0.778 & 0.558 & 0.745 & 0.759 & 0.684 & 0.698 & 0.788 & 0.588 \\
Ex-Jaccard & 0.804 & 0.654 & 0.509 & 0.529 & 0.688 & 0.435 & 0.581 & 0.629 & 0.494 & 0.493 & 0.641 & 0.429 \\
Manhattan & 0.691 & 0.511 & 0.356 & 0.358 & 0.554 & 0.307 & 0.490 & 0.544 & 0.370 & 0.324 & 0.536 & 0.339 \\
KLD & 0.482 & 0.442 & 0.186 & 0.164 & 0.402 & 0.168 & 0.397 & 0.348 & 0.295 & 0.204 & 0.472 & 0.280 \\
SMTP & 0.862 & 0.725 & 0.602 & 0.623 & 0.760 & 0.518 & 0.772 & 0.805 & 0.692 & 0.707 & 0.794 & 0.614 \\
BLAB-SM & 0.870 & 0.717 & 0.586 & 0.617 & 0.751 & 0.508 & 0.776 & 0.828 & 0.693 & 0.712 & 0.795 & 0.625 \\
\hline
\end{tabular}




\section{Table $\mathbf{1 5}$ (on next page)}

Table 15. Purity (mostly known as "Accuracy") - K-means performance

Table 15. Purity (mostly known as "Accuracy") - K-means performance 
1

2

3

4

\begin{tabular}{ccccccc}
$\begin{array}{c}\text { Similarity } \\
\text { measure/ Metric }\end{array}$ & \multicolumn{2}{c}{$\mathrm{K}=5$} & \multicolumn{2}{c}{$\mathrm{K}=10$} & \multicolumn{2}{c}{ K= Number of classes } \\
& $\begin{array}{c}\text { Reuters }-18308 \\
\text { features }\end{array}$ & $\begin{array}{c}\text { Web-KB }- \\
33025 \text { features }\end{array}$ & $\begin{array}{c}\text { Reuters }-18308 \\
\text { features }\end{array}$ & $\begin{array}{c}\text { Web-KB - } \\
33025 \text { features }\end{array}$ & $\begin{array}{c}\text { Reuters }-18308 \\
\text { features }(\mathrm{K}=4)\end{array}$ & $\begin{array}{c}\text { Web-KB }-33025 \\
\text { features }(\mathrm{K}=8)\end{array}$ \\
Euclidean & 0.6348979 & 0.6701596 & 0.7467169 & 0.6244344 & 0.6376284 & 0.6480114 \\
Cosine & 0.6200751 & 0.6546797 & 0.6641529 & 0.6263396 & 0.6727344 & 0.6225292 \\
Jaccard & 0.55571447 & 0.6468207 & 0.6736445 & 0.6663491 & 0.6222858 & 0.6320553 \\
Ex-Jaccard & 0.5942478 & 0.6434865 & 0.6970484 & 0.6261014 & 0.6358080 & 0.6618242 \\
KLD & 0.6091535 & 0.6170517 & 0.7253933 & 0.6477733 & 0.6618125 & 0.6525363 \\
Manhattan & 0.5648979 & 0.5901565 & 0.6368767 & 0.5845236 & 0.6076209 & 0.6281809 \\
BLAB-SM & 0.6313405 & 0.6037151 & 0.6965284 & 0.6954036 & 0.6459498 & 0.63824720 \\
SMTP & 0.6261864 & 0.6408668 & 0.6885970 & 0.6356275 & 0.6374983 & 0.63372231
\end{tabular}




\section{Table $\mathbf{1 6}$ (on next page)}

Table 16. Entropy - K-means performance

Table 16. Entropy - K-means performance 
1

2

3

4

\begin{tabular}{ccccc}
$\begin{array}{c}\text { Similarity } \\
\text { measure/ Metric }\end{array}$ & \multicolumn{2}{c}{$\mathrm{K}=5$} & \multicolumn{2}{c}{$\mathrm{K}=10$} \\
& $\begin{array}{c}\text { Reuters }-18308 \\
\text { features }\end{array}$ & $\begin{array}{c}\text { Web-KB - } \\
33025 \text { features }\end{array}$ & $\begin{array}{c}\text { Reuters }-18308 \\
\text { features }\end{array}$ & $\begin{array}{c}\text { Web-KB - } \\
33025 \text { features }\end{array}$ \\
Euclidean & 0.4181014 & 0.6083767 & 0.3324207 & 0.6338744 \\
Cosine & 0.5079926 & 0.6226576 & 0.3728674 & 0.6445081 \\
Jaccard & 0.4551138 & 0.6505793 & 0.4093773 & 0.6063160 \\
Ex-Jaccard & 0.4210397 & 0.6307335 & 0.3478137 & 0.6219528 \\
KLD & 0.5174439 & 0.6295514 & 0.3337954 & 0.6137706 \\
Manhattan & 0.4481032 & 0.6387609 & 0.3225643 & 0.6034765 \\
BLAB-SM & 0.4941582 & 0.6273114 & 0.3253195 & 0.5561895 \\
SMTP & 0.4647862 & 0.6486512 & 0.3147964 & 0.5373072
\end{tabular}

Table 16. Entropy - K-means performance

\begin{tabular}{cc}
\multicolumn{2}{c}{ K= Number of classes } \\
$\begin{array}{c}\text { Reuters }-18308 \\
\text { features }(\mathrm{K}=4)\end{array}$ & $\begin{array}{c}\text { Web-KB }-33025 \\
\text { features }(\mathrm{K}=8)\end{array}$ \\
0.4066976 & 0.6489474 \\
0.3673066 & 0.6287172 \\
0.4044194 & 0.6138461 \\
0.4078592 & 0.6325090 \\
0.3724656 & 0.6335062 \\
0.3866542 & 0.6089534 \\
0.4045278 & 0.6356724 \\
0.4038267 & 0.6341636
\end{tabular}




\section{Table $\mathbf{1 7}$ (on next page)}

Table 17. Measure Stability Status

Table 17. Measure Stability Status 
1

2

Table 17. Measure Stability Status

3

\begin{tabular}{|c|c|c|c|c|c|c|c|c|c|c|c|c|c|c|}
\hline \multirow{2}{*}{ NF/Measure } & \multicolumn{2}{|c|}{ Euclidean } & \multicolumn{2}{|c|}{ Cosine } & \multicolumn{2}{|c|}{ Ex-Jaccard } & \multicolumn{2}{|c|}{ Manhattan } & \multicolumn{2}{|c|}{ kullback Leibler } & \multicolumn{2}{|c|}{ SMTP } & \multicolumn{2}{|c|}{ BLAB-SM } \\
\hline & $\mathrm{R}$ & $\mathrm{W}$ & $\mathrm{R}$ & $\mathrm{W}$ & $\mathrm{R}$ & $\mathrm{W}$ & $\mathrm{R}$ & $\mathrm{W}$ & $\mathrm{R}$ & $\mathrm{W}$ & $\mathrm{R}$ & $\mathrm{W}$ & $\mathrm{R}$ & $\mathrm{W}$ \\
\hline 10 & & 3 & & 2 & & & 3 & 3 & 3 & 1 & & & 3 & \\
\hline 50 & 2 & & 1 & 3 & & & 2 & & & & 1 & 3 & 1 & 3 \\
\hline 100 & 2 & & & 3 & & & 2 & & & & 3 & 3 & 1 & 3 \\
\hline 200 & & & 3 & 3 & & & & & & & 3 & 3 & 3 & 3 \\
\hline 350 & & & 3 & 3 & & & & & & & 3 & 3 & 3 & 3 \\
\hline 3000 & & & 3 & 3 & & & & & & & 3 & 3 & 3 & 3 \\
\hline 6000 & & & 2 & 3 & 1 & & & & & & 3 & 3 & 3 & 3 \\
\hline All features & 2 & 3 & 2 & 3 & 1 & & & & & & 3 & 3 & 1 & 3 \\
\hline Sum & 6 & 6 & 14 & 23 & 2 & & 7 & 3 & 3 & 1 & 19 & 21 & 18 & 21 \\
\hline Stable points & & & 3 & 9 & 4 & & & 11 & & & 4 & & & \\
\hline
\end{tabular}




\section{Table 18 (on next page)}

Table 18. Reuters - Statistical Significance of the experimental results of BLAB-SM against all measures

Table 18. Reuters - Statistical Significance of the experimental results of BLAB-SM against all measures 
Table 18. Reuters - Statistical Significance of the experimental results of BLAB-SM against all measures

\begin{tabular}{|l|l|l|l|l|}
\hline Measure & MAE & MSE & Std Error & Confidence Interval \\
\hline Manhattan & 0.473236691 & 0.22496147 & 0.0040998006 & $0.51855962-0.53496698$ \\
\hline Euclidean & 0.117193134 & 0.01375278 & 0.0005560327 & $0.88169424-0.88391948$ \\
\hline Cosine & 0.114459829 & 0.01312222 & 0.0005939627 & $0.88435165-0.88672868$ \\
\hline Jaccard & 0.497886917 & 0.25102953 & 0.0072320509 & $0.48764178-0.51658438$ \\
\hline KLD & 0.496776287 & 0.24930169 & 0.0064743325 & $0.49026860-0.51617882$ \\
\hline SMTP & $\mathbf{0 . 0 9 5 4 8 5 3 5 9}$ & $\mathbf{0 . 0 0 9 3 1 8 6 0}$ & 0.0018312414 & $\mathbf{0 . 9 0 0 8 5 0 3 3 - ~ 0 . 9 0 8 1 7 8 9 4}$ \\
\hline Ex-Jaccard & $\mathbf{0 . 0 9 1 5 1 1 6 1 1}$ & $\mathbf{0 . 0 0 8 4 5 2 7 5}$ & 0.0011429639 & $\mathbf{0 . 9 0 6 2 0 1 3 2 - ~ 0 . 9 1 0 7 7 5 4 5}$ \\
\hline BLAB-SM & $\mathbf{0 . 0 9 9 1 1 1 7 8 5}$ & $\mathbf{0 . 0 1 0 0 6 0 8 1}$ & 0.0019902300 & $\mathbf{0 . 8 9 6 9 0 5 7 7 - ~ 0 . 9 0 4 8 7 0 6 5}$ \\
\hline
\end{tabular}




\section{Table $\mathbf{1 9}$ (on next page)}

Table 19. Reuters -Statistical significance with non-parametric Wilcoxon for BLAB-SM against all measures

Table 19. Reuters -Statistical significance with non-parametric Wilcoxon for BLAB-SM against all measures 
Table 19. Reuters -Statistical significance with non-parametric Wilcoxon for BLAB-SM against all measures

\begin{tabular}{|l|l|l|}
\hline Measure & Wilcoxon Z Value & Wilcoxon P Value \\
\hline Manhattan & -6.7359808238 & 0.0 \\
\hline Euclidean & -6.4488297578 & 0.00000000001 \\
\hline Cosine & -5.6390451991 & 0.0000000171 \\
\hline Jaccard & -6.7365741193 & 0.0 \\
\hline KLD & -6.7365512974 & 0.0 \\
\hline SMTP & -5.9849832403 & 0.0000000022 \\
\hline Ex-Jaccard & -4.9985622992 & 0.0000005776 \\
\hline BLAB-SM & Nan & nan \\
\hline
\end{tabular}




\section{Table 20 (on next page)}

Table 20. Reuters -Statistical significance with paired t-test (DF is degrees of freedom) for BLAB-SM against all measures

Table 20. Reuters -Statistical significance with paired t-test (DF is degrees of freedom) for BLAB-SM against all measures 
Table 20. Reuters -Statistical significance with paired t-test (DF is degrees of freedom) for BLAB-SM against all measures

\begin{tabular}{|l|l|l|l|}
\hline Measure & t-score & tTest p-value & DF \\
\hline Manhattan & -124.5610100138 & 0.0 & 59.0 \\
\hline Euclidean & -8.2792339235 & 0.0 & 59.0 \\
\hline Cosine & -6.8900087347 & 0.0000000042 & 59.0 \\
\hline Jaccard & -49.7455369193 & 0.0 & 59.0 \\
\hline KLD & -54.7808677782 & 0.0 & 59.0 \\
\hline SMTP & 10.3197557538 & 0.0 & 59.0 \\
\hline Ex-Jaccard & 5.6215858102 & 0.000000543 & 59.0 \\
\hline BLAB-SM & Nan & nan & 59.0 \\
\hline
\end{tabular}




\section{Table 21 (on next page)}

Table 21. Web-KB - Significance Test of BLAB-SM against all measures

Table 21. Web-KB - Significance Test of BLAB-SM against all measures 
Table 21. Web-KB - Significance Test of BLAB-SM against all measures

\begin{tabular}{|l|l|l|l|l|}
\hline Measure & MAE & MSE & Std Error & Confidence Interval \\
\hline Manhattan & 0.602047015 & $\mathbf{0 . 3 6 2 9 6 2 2 7 1}$ & 0.002891546 & $0.39216701-0.40373895$ \\
\hline Euclidean & 0.239183835 & 0.058129364 & 0.003916753 & $0.75297875-0.76865356$ \\
\hline Cosine & 0.238087691 & 0.057424802 & 0.003509637 & $0.75488953-0.76893507$ \\
\hline Jaccard & 0.776096143 & 0.602833150 & 0.002909542 & $0.21808187-0.22972583$ \\
\hline KLD & 0.777548864 & 0.605136971 & 0.003040655 & $0.21636679-0.22853547$ \\
\hline SMTP & $\mathbf{0 . 1 9 5 6 6 8 2 5 1}$ & $\mathbf{0 . 0 3 8 4 1 3 1 0 4}$ & $\mathbf{0 . 0 0 1 4 5 5 1 0 3}$ & $\mathbf{0 . 8 0 1 4 2 0 0 9} \mathbf{- 0 . 8 0 7 2 4 3 4 0}$ \\
\hline Ex-Jaccard & 0.240649762 & 0.058737734 & 0.003709056 & $0.75192843-0.76677204$ \\
\hline BLAB-SM & $\mathbf{0 . 1 8 6 2 8 3 0 6 8}$ & $\mathbf{0 . 0 3 4 8 0 1 8 4 9}$ & $\mathbf{0 . 0 0 1 2 9 4 0 1 2}$ & $\mathbf{0 . 8 1 1 1 2 7 6 1} \mathbf{- 0 . 8 1 6 3 0 6 2 4}$ \\
\hline
\end{tabular}

2 


\section{Table 22 (on next page)}

Table 22. Web-KB - Statistical significance with non-parametric Wilcoxon for BLAB-SM against all measures

Table 22. Web-KB - Statistical significance with non-parametric Wilcoxon for BLAB-SM against all measures 
Table 22. Web-KB - Statistical significance with non-parametric Wilcoxon for BLAB-SM against all measures

\begin{tabular}{|l|l|c|}
\hline Measure & Wilcoxon Z Value & Wilcoxon P Value \\
\hline Manhattan & -6.7365284757 & 0.0 \\
\hline Euclidean & -6.7359351922 & 0.0 \\
\hline Cosine & -6.7358895615 & 0.0 \\
\hline Jaccard & -6.7368936511 & 0.0 \\
\hline KLD & -6.7368936511 & 0.0 \\
\hline SMTP & -6.213256068 & 0.0000000005 \\
\hline Ex-Jaccard & -6.7358667465 & 0.0 \\
\hline BLAB-SM & nan & nan \\
\hline
\end{tabular}

2 


\section{Table 23 (on next page)}

Table 23. Web-KB - Statistical significance with paired t-test (DF stands for degrees of freedom)

Table 23. Web-KB - Statistical significance with paired t-test (DF stands for degrees of freedom) 
Table 23. Web-KB - Statistical significance with paired t-test (DF stands for degrees of freedom)

\begin{tabular}{|l|l|l|l|}
\hline Measure & t-score & tTest p-value & DF \\
\hline Manhattan & -116.7083551117 & 0.0 & 59.0 \\
\hline Euclidean & -13.9243714309 & 0.0 & 59.0 \\
\hline Cosine & -15.2824025577 & 0.0 & 59.0 \\
\hline Jaccard & -158.01549932 & 0.0 & 59.0 \\
\hline KLD & -153.0210104997 & 0.0 & 59.0 \\
\hline SMTP & -11.223396507 & 0.0 & 59.0 \\
\hline Ex-Jaccard & -14.5841724659 & 0.0 & 59.0 \\
\hline BLAB-SM & nan & nan & 59.0 \\
\hline
\end{tabular}

2

3 


\section{Table 24(on next page)}

Table 24. Clustering Process - Points and Rank of Similarity Measures

Table 24. Clustering Process - Points and Rank of Similarity Measures 
1

2

3

Table 18. Clustering Process - Points and Rank of Similarity Measures

\begin{tabular}{ccc}
\hline Similarity Measure & Points (out of 12) & Rank \\
\hline Euclidean & 6 & 1 \\
Cosine & 5 & 2 \\
Jaccard & 3 & - \\
Ex-Jaccard & 3 & - \\
KLD & 5 & 2 \\
Manhattan & 4 & 3 \\
BLAB-SM & 6 & 1 \\
SMTP & 4 & 2 \\
\hline
\end{tabular}

4 


\section{Table $\mathbf{2 5}$ (on next page)}

Table 25. Comparison of Classification Run Time (in Minutes) of BLAB-SM against all Measures on both Datasets

Table 25. Comparison of Classification Run Time (in Minutes) of BLAB-SM against all Measures on both Datasets 
1

2

3

4

Table 19. Comparison of Classification Run Time (in Minutes) of BLAB-SM against all Measures on both Datasets

\begin{tabular}{ccccc}
\hline Measure/ Dataset & Reuters (18308 features) & Improvement Rate & Web-KB (33025 features) & Improvement Rate \\
\hline BLAB-SM & 29 & - & 23 & - \\
Cosine & 58.28 & $50.24 \%$ & 26.37 & $12.78 \%$ \\
SMTP & 805 & $96.40 \%$ & 337 & $93.18 \%$ \\
Euclidean & 34.17 & $15.13 \%$ & 20.42 & $-11.22 \%$ \\
Manhattan & 49.14 & $40.98 \%$ & 23.10 & $0.44 \%$ \\
Jaccard & 38.19 & $24.06 \%$ & 18.24 & $-20.69 \%$ \\
kullback Leibler & 112.35 & $74.19 \%$ & 59.37 & $61.02 \%$ \\
\hline
\end{tabular}

5 